\title{
15. GEOCHEMISTRY OF PORE WATER AND SEDIMENTS RECOVERED FROM THE EXMOUTH PLATEAU1
}

\author{
Eric H. De Carlo²
}

\begin{abstract}
During Leg 122, a transect was drilled across the Wombat Plateau, a marginal spur of the Exmouth Plateau, complemented by two sites on the Exmouth Plateau proper. In this report, pore-water analyses for major seawater constituents, alkalinity, $\mathrm{Ba}, \mathrm{Fe}, \mathrm{Mn}, \mathrm{Li}, \mathrm{Sr}, \mathrm{Rb}$, and silica are presented. Large gradients in the pore-water profiles provide evidence of complex sediment/pore-water interactions associated with carbonate and silica diagenesis and the formation of authigenic minerals. Diffusion affects pore-water profiles but differs considerably from site to site. Advection of freshwater, probably of continental origin, helps maintain negative $\mathrm{Cl}$ and salinity gradients deep within the sediments of the Exmouth Plateau.
\end{abstract}

\section{INTRODUCTION}

Six sites in passive continental margin sediments of the Exmouth and Wombat plateaus, northwest Australia, were drilled during Ocean Drilling Program (ODP) Leg 122 (Fig. 1). Pore water was sampled in Carnian to Quaternary age sediments comprised of continental and shallow marine clastics, shallow and deep marine carbonates, and authigenic minerals. To the best of my knowledge Leg 122 is the first deep ocean drilling leg to have sampled pore waters from Triassic sediments in the Southern Hemisphere.

The Exmouth Plateau is a deeply subsided fragment of rifted continental crust. The plateau began to assume its current configuration during the Late Triassic, when rifting between northwestern Australia, India, and other portions of East Gondwana began (Exon et al., 1982). Breakup and separation of the plateau occurred during the Jurassic to Early Cretaceous and was followed by the deposition of several kilometers of marine sediments. The Wombat Plateau, located at approximately $17^{\circ} \mathrm{S}, 115^{\circ} \mathrm{E}$ (Fig. 1), is a marginal spur of the Exmouth. A detailed description of the regional geology and tectonic evolution of the Exmouth and Wombat plateaus is given by Haq, von Rad, O'Connell, et al. (1990) and von Rad et al. (this volume).

Shipboard pore-water analyses of salinity, $\mathrm{pH}$, alkalinity, $\mathrm{Ca}, \mathrm{Cl}, \mathrm{Mg}$, sulfate, and silica concentrations, and shorebased determinations of $\mathrm{Li}, \mathrm{Na}, \mathrm{K}, \mathrm{Rb}, \mathrm{Sr}, \mathrm{Ba}, \mathrm{Mn}$, and $\mathrm{Fe}$ concentrations in pore water, as well as the concentrations of $\mathrm{Li}, \mathrm{Na}, \mathrm{K}, \mathrm{Mg}, \mathrm{Ca}, \mathrm{Sr}, \mathrm{Ba}, \mathrm{Al}, \mathrm{Si}, \mathrm{Ti}, \mathrm{Fe}$, and $\mathrm{Mn}$ in the sediment, are presented. Pore-water profiles are discussed in terms of sediment diagenesis and in relation to the lithology of the associated sediments.

Elemental compositions of the sediments are not strongly affected by diagenesis. Changes in elemental abundances in the bulk sediment are primarily related to changes in the dominant mineralogy of the sediments and are indicative of changes in the depositional setting of the Exmouth and Wombat plateaus over geologic time. Changes in pore-water elemental abundances, on the other hand, reflect diagenetic processes.

\footnotetext{
${ }^{1}$ von Rad, U., Haq, B. U., et al., 1992. Proc. ODP, Sci. Results, 122: College Station, TX (Ocean Drilling Program).

2 Department of Oceanography, School of Ocean and Earth Sciences and Technology, 1000 Pope Rd., University of Hawaii, Honolulu, Hawaii 96822 U.S.A.
}

\section{METHODS}

\section{Pore Water}

Interstitial water was obtained by shipboard squeezing of whole-round $(5-10 \mathrm{~cm})$ cores at room temperature with a stainless steel press (Manheim and Sayles, 1974); samples were then filtered and stored for subsequent analyses. Alkalinity, $\mathrm{pH}$, and salinity were determined immediately after sample collection. An in-situ harpoon (Barnes) water sampler with a temperature probe was deployed five times. Methods employed at sea have been described by Gieskes (1974) and Gieskes and Peretsman (1986) as well as in the "Explanatory Notes" chapter of the Initial Reports (Haq, von Rad, O'Connell, et al., 1990). Standards calibrated against or prepared from International Association of Physical Scientists Organizations (IAPSO) were used in all shipboard determinations. Acidified splits of samples in glass vials were analyzed by flame atomic absorption-emission $(\mathrm{K}, \mathrm{Li})$, furnace atomic absorption (Rb), and inductively coupled plasma atomic emission ( $\mathrm{Ba}, \mathrm{Fe}, \mathrm{Mn}, \mathrm{Sr}$ ) spectrometries. Selected acidified (alkalinity) splits stored in plastic were also analyzed for $\mathrm{Ba}$ and compared to the glass vial split analyses; this was necessary because erroneously high $\mathrm{Ba}$ concentrations have been observed in samples stored in borosilicate glass (M. Mottl, pers. comm., 1989). Commercial spectroscopic standards were used for calibration and IAPSO seawater used as a check solution in all shore-based determinations. Sodium was determined by charge balance.

\section{Sediments}

Portions of the whole-round squeeze cakes and other solid samples of special interest were rinsed with distilled deionized water to remove sea salts. The sediments were freeze-dried and ground to pass $100-\mu \mathrm{m}$ mesh standard sieves prior to further treatment. Bulk mineralogy was determined by X-ray diffractometry prior to elemental analysis. Results of the mineralogical analyses are presented elsewhere by Wilkens et al. (this volume).

Splits of all samples were dried at $110^{\circ} \mathrm{C}$ and dissolved in microwave digestion vessels. Carbonate-rich sediments were solubilized with $\mathrm{HCl}$, whereas those containing significant amounts of aluminosilicates required a mixture of $\mathrm{HCl}, \mathrm{HF}$, and $\mathrm{HNO}_{3}$. The microwave techniques employed in this work 


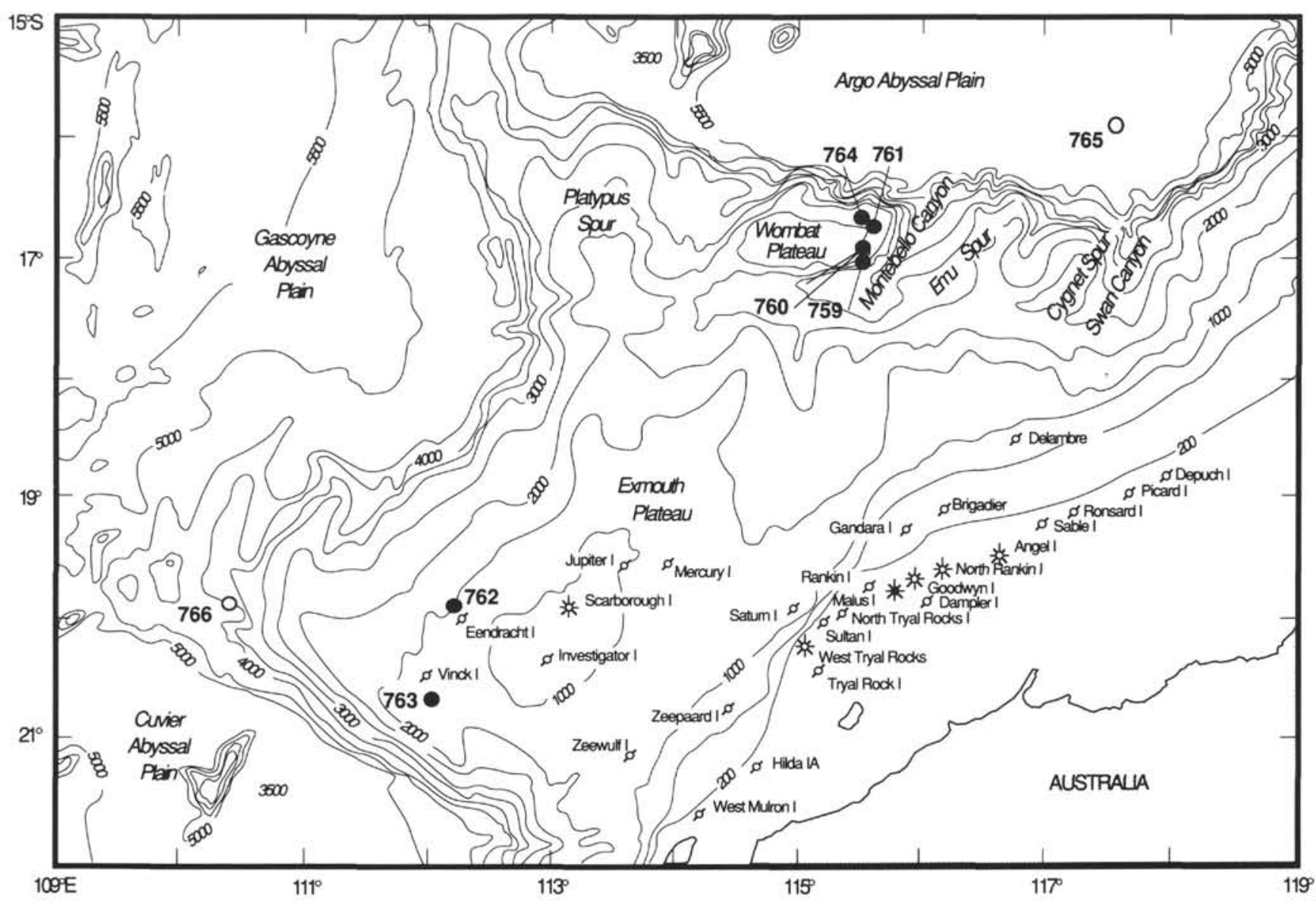

Figure 1. Area location map of the Exmouth Plateau, northwest Australia (after Haq, von Rad, O'Connell, et al., 1989).

are modifications of previously described methods (Kingston and Jassie, 1988; De Carlo, 1990). The accuracy of spectroscopic determinations was verified by the analysis of standard geological materials.

\section{RESULTS}

\section{Wombat Plateau Sites}

Variations in pore-water constituents with depth for Sites 759 through 761 are shown in Figure 2. Data from Site 764 are not presented, as only two pore-water samples were recovered at this site. A summary compilation of pore-water data for Leg 122 sites is presented in Table 1. Results of the bulk elemental analyses of sediments are presented in Table 2 . These data are primarily presented to complement the mineralogical analyses given by Wilkens et al. (this volume). The composition of the sediments will be discussed only in terms of its effect on the pore-water composition.

Major-element concentrations generally exhibit greater variability with increasing depth downhole at Sites 759 and 760 than at Site 761. Similar trends are also observed for other pore-water constituents with the notable exception of $\mathrm{Fe}, \mathrm{Mn}$, and silica. The concentrations of $\mathrm{Mg}, \mathrm{K}$, and sulfate generally decrease in a regular fashion downhole (Fig. 2) to approximately $20 \%$ of the seawater value at depth. Strontium and $\mathrm{Ba}$ concentrations display an opposite behavior and increase downhole. The $\mathrm{Ca}$ profile, unlike those of most major porewater constituents, is rather monotonous at Sites 759 and 761 , but concentrations increase by nearly a factor of two in the first 200 meters below seafloor (mbsf) at Site 760 . Dissolved
$\mathrm{Rb}$ concentrations generally decrease downhole, although the data show significant scatter at Site 760 . The Rb profiles appear similar to those of $\mathrm{K}$.

Dissolved silica profiles are more complex than those of the major pore-water constituents. Typically, a large initial increase above the seawater concentration occurs just below the mudline (Fig. 2) that is followed by a sharp negative gradient between approximately 20 and 50 mbsf. Below this depth dissolved silica concentrations exhibit considerable scatter; large variations are observed primarily at Site 761 .

Dissolved $\mathrm{Fe}$ increases sharply in the first $25 \mathrm{~m}$ of the sediment then decreases to $1-2 \mu \mathrm{mol} / \mathrm{L}$ at $100 \mathrm{mbsf}$. Between 100 and 150 mbsf significant increases in dissolved $\mathrm{Fe}$ are observed at Sites 759 and 760 , whereas only a small increase occurs at Site 761. Elevated dissolved Mn concentrations immediately below the sediment/seawater interface decrease to less than $2 \mu \mathrm{mol} / \mathrm{L}$ by $25 \mathrm{mbsf}$, increase to maxima between 50 and 80 mbsf, then return to lower concentrations at depth. A monotonous Mn profile is observed only at Site 759.

\section{Sites 762 and 763}

Unlike the sedimentary sequences encountered at Sites 759,760 , and 761 , fairly continuous sequences comprised of Quaternary through Berriasian sediments were recovered at Sites 762 and 763 (Haq, von Rad, O'Connell, et al., 1990). Much of the sediment is carbonate-rich, but grades into clay and siliciclastic-rich sediments below 800 and $500 \mathrm{mbsf}$ at the two sites, respectively. Excellent core recovery led to the collection of 34 pore-water samples at Site 762 and 41 at Site 763. The sedimentary sequences at Sites 762 and 763 are 


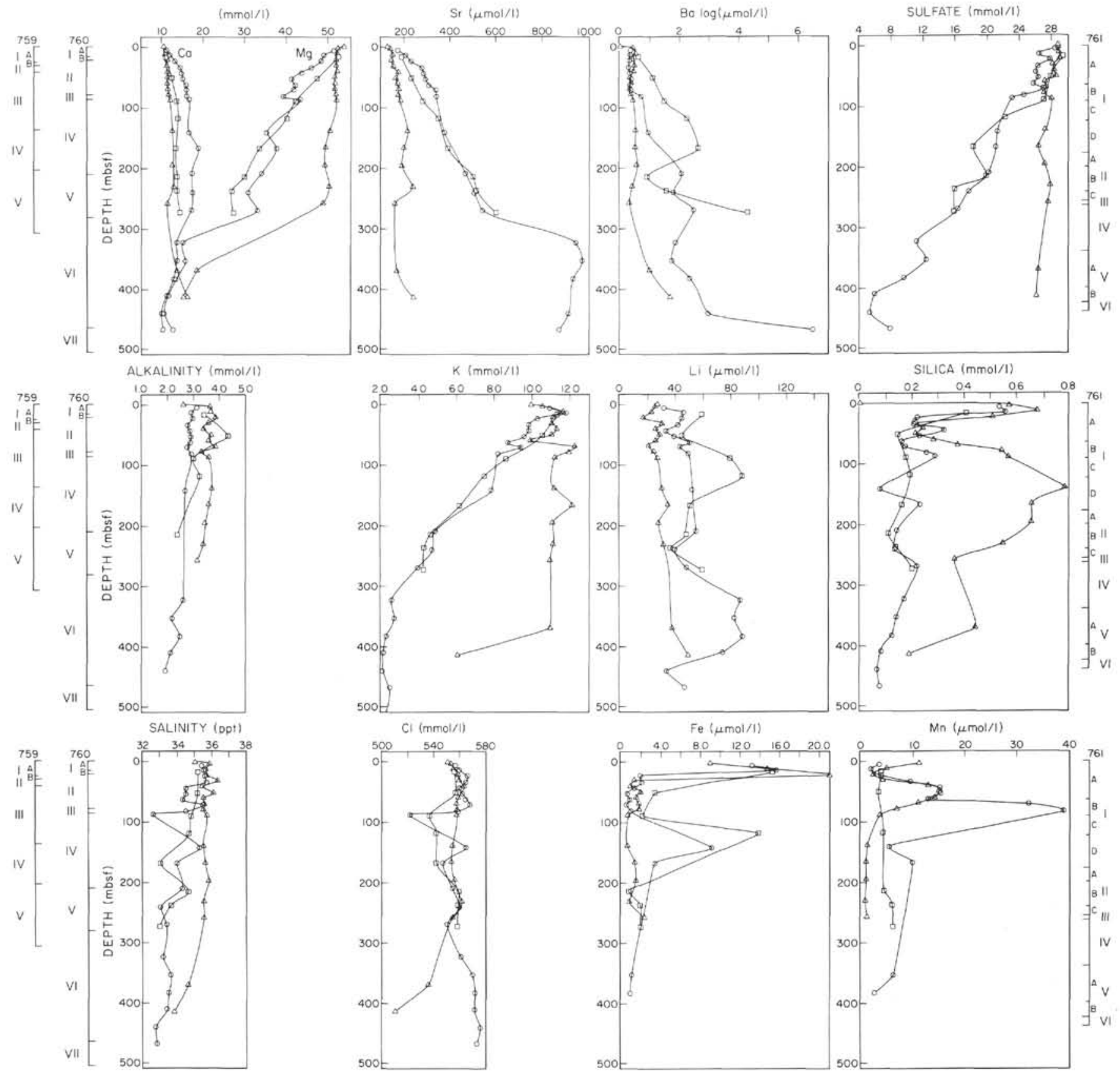

Figure 2. Wombat Plateau sites: pore-water concentrations as a function of depth with lithological units indicated to the left. Squares $=$ Site 759 , circles $=$ Site 760 , and triangles $=$ Site 761 .

similar with the exception of a major hiatus from the early Maestrichtian to middle Eocene at Site 763. Thus, the sediments from Site 763 provided the opportunity to study an expanded section of Turonian to Berriasian sediments unavailable at Site 762 .

Nearly all pore-water profiles at Sites 762 and 763 (Fig. 3) are characterized by significant concentration gradients. Other differences between the Wombat Plateau sites (Fig. 2) and Sites 762 and 763 (Fig. 3) are the steeper overall dissolved $\mathrm{Li}$ gradient, and much steeper $\mathrm{Sr}$ and sulfate pore-water gradients in the uppermost sections of the latter.

Alkalinity profiles at Sites 762 and 763 are markedly different from one another. At Site 762 alkalinity gradually increases with depth for the first $370 \mathrm{~m}$ except for a sharp increase to $9.6 \mathrm{mmol} / \mathrm{L}$ at $404 \mathrm{mbsf}$. Below this depth, the alkalinity decreases downhole, except for a single high measurement at 887 mbsf. The alkalinity at Site 763 , however, is characterized by considerable variability in the first $150 \mathrm{mbsf}$, below which a broad, well-defined maximum peaking at 320 mbsf is observed. Further downhole alkalinity fluctuates between 2 and $4 \mathrm{mmol} / \mathrm{L}$.

Dissolved silica exhibits trends that are similar to those observed at Site 761, providing evidence of several reaction zones within the sediments. The trace element profiles are also quite variable. Dissolved $\mathrm{Mn}$ and $\mathrm{Fe}$ exhibit complex profiles at both Exmouth Plateau sites. Some similarities exist with the profiles at Site 761 (Fig. 2) and are particularly evident in the uppermost $200 \mathrm{~m}$ of the sediments. Three and 
Table 1. Composition of pore water from Leg 122.

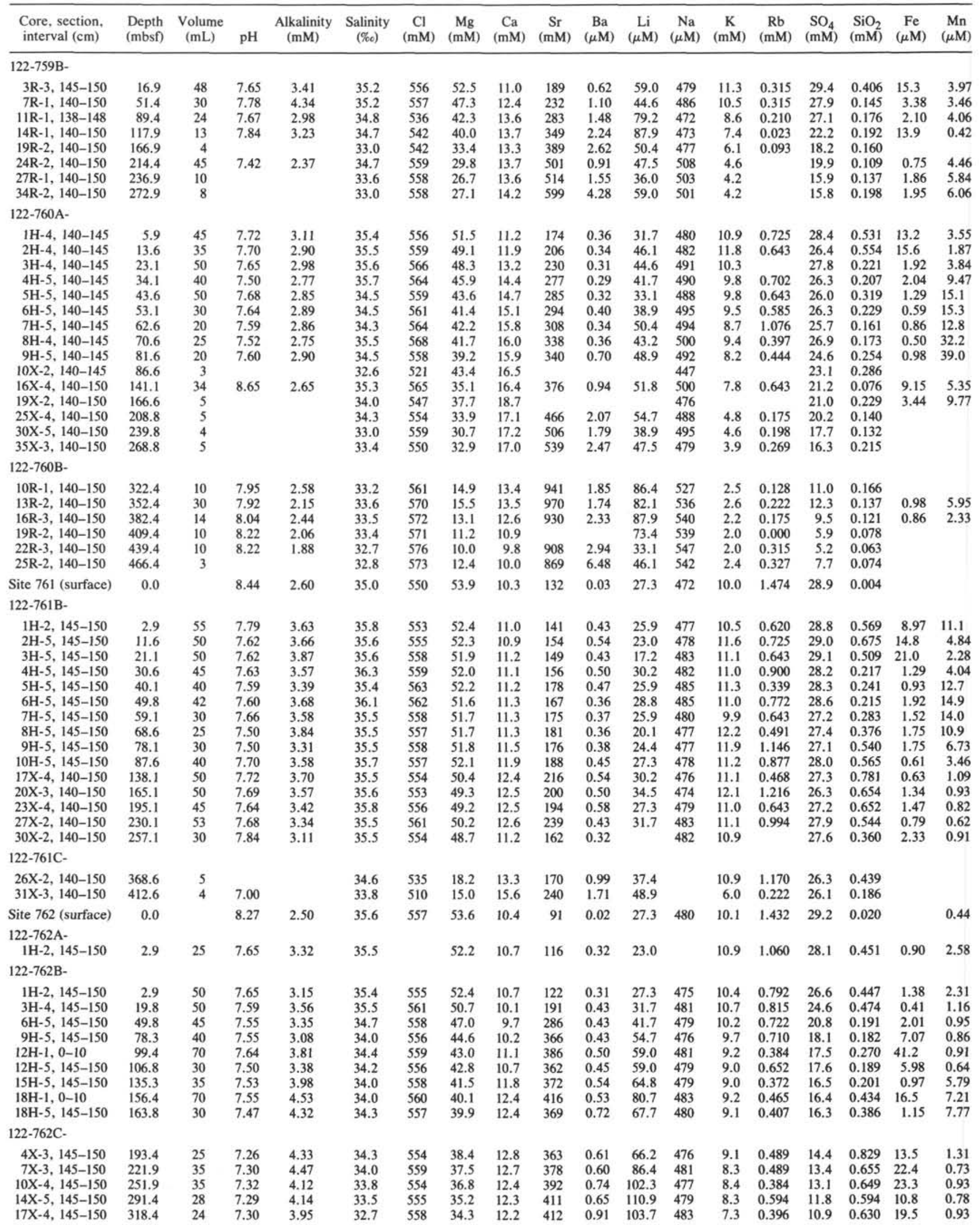


Table 1 (continued).

\begin{tabular}{|c|c|c|c|c|c|c|c|c|c|c|c|c|c|c|c|c|c|c|}
\hline $\begin{array}{l}\text { Core, section, } \\
\text { interval }(\mathrm{cm})\end{array}$ & $\begin{array}{l}\text { Depth } \\
\text { (mbsf) }\end{array}$ & $\begin{array}{c}\text { Volume } \\
(\mathrm{mL})\end{array}$ & pH & $\begin{array}{l}\text { Alkalinity } \\
(\mathrm{mM})\end{array}$ & $\begin{array}{l}\text { Salinity } \\
\left(\% \sigma_{0}\right)\end{array}$ & $\underset{(\mathrm{mM})}{\mathrm{Cl}}$ & $\underset{(\mathrm{mM})}{\mathrm{Mg}}$ & $\underset{(\mathrm{mM})}{\mathrm{Ca}}$ & $\underset{(\mathrm{mM})}{\mathrm{Sr}}$ & $\underset{(\mu \mathrm{M})}{\mathrm{Ba}}$ & $\begin{array}{c}\mathrm{Li} \\
(\mu \mathrm{M})\end{array}$ & $\begin{array}{c}\mathrm{Na} \\
(\mu \mathrm{M})\end{array}$ & $\underset{(\mathrm{mM})}{\mathrm{K}}$ & $\underset{(\mathrm{mM})}{\mathrm{Rb}}$ & $\begin{array}{l}\mathrm{SO}_{4} \\
(\mathrm{mM})\end{array}$ & $\begin{array}{l}\mathrm{SiO}_{2} \\
(\mathrm{mM})\end{array}$ & $\underset{(\mu \mathrm{M})}{\mathrm{Fe}}$ & $\underset{(\mu \mathrm{M})}{\mathrm{Mn}}$ \\
\hline $20 \mathrm{X}-2,145-150$ & 343.9 & 28 & 7.39 & 5.16 & 33.0 & 559 & 32.9 & 12.1 & 459 & 0.76 & 144.1 & 486 & 6.8 & 0.419 & 9.6 & 0.759 & 8.52 & 0. \\
\hline $23 \mathrm{X}-2,140-150$ & 372.4 & 17 & 25 & 4.09 & 33.2 & 556 & 32.8 & 13.0 & 422 & 1.09 & 106.6 & 475 & 6.5 & 0.302 & 7.0 & 0.723 & 0.32 & 0.71 \\
\hline $26 \mathrm{X}-4,140-150$ & 403.9 & 34 & 6.90 & 9.63 & 33.2 & 555 & 25.2 & 18.2 & 425 & 2.38 & 126.8 & 492 & 5.6 & 0.244 & 10.2 & 1.126 & 0.84 & 0.58 \\
\hline $29 \mathrm{X}-2,140-150$ & 424.4 & 19 & 7.22 & 4.73 & 32.9 & 557 & 27.5 & 15.4 & 452 & 2.35 & 122.4 & 485 & 5.7 & 0.198 & 7.7 & 0.838 & 3.03 & 0.75 \\
\hline $32 \mathrm{X}-2,140-150$ & 452.9 & 15 & 7.36 & 5.37 & 32.5 & 551 & 27.1 & 14.5 & 560 & 1.97 & 113.8 & 482 & 5.4 & & 7.4 & 0.696 & 0.47 & 0.69 \\
\hline $35 \mathrm{X}-1,140-150$ & 479.9 & 13 & 7.17 & 4.90 & 32.5 & 551 & 25.2 & 16.1 & 498 & 3.37 & 134.0 & 484 & 5.0 & 0.256 & 7.8 & 0.857 & 1.63 & 1.07 \\
\hline $44 X-4,140-150$ & 569.9 & 34 & 7.26 & 4.47 & 31.7 & 545 & 19.9 & 15.2 & 531 & 42.23 & 144.1 & 481 & 3.6 & 0.069 & 3.1 & 0.768 & 0.52 & 0.67 \\
\hline $47 X-5,140-150$ & 599.9 & 28 & 7.32 & 4. & 31.6 & 542 & 20.6 & 13.8 & 506 & 9.83 & 132.5 & 479 & 3.5 & 0.198 & 3.2 & 0.340 & 2.44 & 0.78 \\
\hline $50 X-2,140-150$ & 623.9 & 9 & 7.24 & 2.24 & 31.0 & 545 & 18.4 & 14.4 & 447 & 124.51 & 123.9 & 481 & 2.7 & 0.116 & 1.6 & 0.225 & & \\
\hline $53 \mathrm{X}-4,140-150$ & 655.4 & 11 & 7.17 & 3.22 & 31.0 & 538 & 17.9 & 14.3 & 517 & 18.78 & 148.4 & 478 & 3.2 & 0.250 & 2.6 & 0.238 & & \\
\hline $56 X-5,140-150$ & 685.4 & 29 & 7.50 & 2.00 & 30.7 & 537 & 16.4 & 12.7 & 452 & 14.05 & 136.9 & 483 & 3.5 & 0.163 & 3.4 & 0.188 & 0.86 & 0.96 \\
\hline $59 \mathrm{X}-4,140-150$ & 712.4 & 34 & 7.44 & 2.57 & 30.5 & 529 & 15.4 & 12.6 & 501 & 22.57 & 138.3 & 481 & 3.5 & 0.221 & 5.0 & 0.250 & 5.41 & 0.49 \\
\hline $62 X-4,140-150$ & 740.9 & 20 & 7.42 & 2.74 & 30.7 & 535 & 15.0 & 13.7 & 410 & 8.81 & 126.8 & 482 & 3.5 & 0.244 & 2.9 & 0.344 & 1.67 & 0.49 \\
\hline $66 \mathrm{X}-4,140-150$ & 770.9 & 30 & 7.37 & 2.87 & 30.4 & 535 & 12.9 & 14.0 & 426 & 60.21 & 131.1 & 484 & 3.0 & 0.244 & 1.8 & 0.427 & 0.82 & 0.40 \\
\hline $72 X-1,140-150$ & 796.4 & 0.5 & & & 30.5 & 526 & & & & & & & & & 2.3 & 0.179 & & \\
\hline $82 X-3,140-150$ & 857.9 & 3 & 7.35 & & 28.2 & 491 & 11.3 & 12.5 & & & & & & & 1.9 & 0.150 & & \\
\hline $86 X-3,14$ & 886.9 & 9 & 7.45 & 6.61 & 28.4 & 502 & 9.4 & 13.1 & 480 & 391.75 & 201.7 & 467 & 2.0 & 0.128 & 2.6 & 0.213 & & \\
\hline $89 X-4,140-150$ & 916.9 & 5 & & & 28.4 & 495 & 11.6 & 12.8 & 476 & 24.53 & 178.7 & 441 & 1.8 & 0.256 & 2.1 & 0.273 & & \\
\hline Site 763 (surface) & & 0.0 & 8.16 & 2.28 & 35.5 & 559 & 54.2 & 10.5 & 92 & 0.07 & 26.6 & 480 & 10.1 & 1.567 & 29.6 & 0.002 & 0.07 & 0.47 \\
\hline \multicolumn{19}{|l|}{$122-763 \mathrm{~A}-$} \\
\hline $1 \mathrm{H}-2,14$ & 2.9 & 50 & 7.59 & 3.36 & 35.8 & 555 & 52.8 & 10.7 & 135 & 0.36 & 41.7 & 476 & 11.3 & 0.351 & 28.0 & 0.489 & 8.52 & 2.66 \\
\hline 150 & 10.8 & 5 & 7.46 & 3.76 & 35.6 & 556 & 50.6 & 10.4 & 207 & 0.91 & 31.7 & 477 & 12.1 & 0.830 & 25.9 & 0.579 & 1.43 & 1.46 \\
\hline $3 \mathrm{H}-4,1$ & 20.3 & 4 & 7.36 & 3. & 35.2 & 556 & 48.4 & 9.8 & 221 & 0.38 & 28.8 & 479 & 10.8 & 0.409 & 23.6 & 0.571 & 1.33 & 0.98 \\
\hline $4 \mathrm{H}-4,1$ & 29.8 & 47 & 7.45 & 4.04 & 35.1 & 559 & 47.0 & 9.4 & 268 & 0.38 & 23.0 & 486 & 10.7 & .526 & 23.8 & 0.450 & 0.81 & 0.98 \\
\hline .150 & 39.3 & 50 & 7.46 & & 34.6 & 556 & 45.2 & 9.0 & 326 & 0.62 & 27.3 & 480 & 10.7 & 0.526 & 19.9 & 0.331 & 4.66 & 0.80 \\
\hline $6 \mathrm{H}-4,1$ & 48.8 & 40 & 7.44 & 3.0 & 34.5 & 556 & 43.4 & 8.8 & 356 & 0.45 & 36.0 & 481 & 10.2 & .725 & 18.9 & 0.253 & 9.65 & 1.11 \\
\hline $7 \mathrm{H}-1$ & 52.4 & 55 & 7.66 & 3.6 & 34.5 & 559 & 43.4 & 9.0 & 357 & 0.06 & 23.0 & 487 & 8.7 & & 19.0 & 0.227 & 10.2 & 0.98 \\
\hline $7 \mathrm{H}-4,1$ & 58.3 & 4 & 7.44 & 3.4 & 34.6 & 559 & 42.1 & 8.9 & 398 & 0.72 & 43.2 & 485 & 9.7 & 0.514 & 17.3 & 0.202 & 9.87 & 0.86 \\
\hline $8 \mathrm{H}-4$ & 67.8 & 40 & 7.41 & 3.3 & 34.3 & 559 & 41.2 & 8.7 & 442 & 1.04 & 47.5 & 485 & 9.5 & 0.468 & 16.6 & 0.180 & 1.20 & 1.00 \\
\hline $9 \mathrm{H}-4,1$ & 77.3 & 38 & 7.41 & 3.1 & 33.9 & 556 & 40.5 & 8.7 & 467 & 0.93 & 41.7 & 483 & 9.6 & 0.456 & 16.4 & 0.167 & 9.20 & 0.66 \\
\hline $10 \mathrm{H}-4,1$ & 86.8 & 3 & 7.46 & 2.9 & 33.8 & 556 & 39.3 & 8.7 & 476 & 1.1 & 43.2 & 482 & 9.8 & 0.315 & 15.3 & 0.159 & 5.55 & 0.58 \\
\hline $11 \mathrm{H}-1,0$ & 90.4 & 65 & .63 & 3.12 & 33.9 & 559 & 39.8 & 8.9 & 500 & 0.4 & 40.3 & 484 & 9.1 & 0.409 & 14.7 & 0.196 & 9.94 & 0.78 \\
\hline $13 \mathrm{H}-4,1$ & 115.3 & 3 & 7.50 & 3 & 33.8 & 556 & 37.9 & 9.0 & 484 & 1.1 & 41.7 & 482 & 8.8 & 0.362 & 13.1 & 0.145 & 4.62 & 0.86 \\
\hline $16 \mathrm{H}-$ & 143.8 & 33 & 7.48 & 3.1 & & 560 & 38 & 8.4 & 481 & 0. & 41.7 & 486 & 9.1 & 2 & 13.7 & 0.151 & 3.49 & 2.52 \\
\hline $17 \mathrm{H}-1,0$ & 147.4 & 6 & 7.63 & 2.73 & 34.2 & 562 & 43.1 & 8.8 & 419 & 0.33 & 40.3 & 492 & 8.9 & 0.327 & 20.9 & 0.098 & 0.75 & 1.29 \\
\hline $19 \mathrm{H}-4,145-150$ & 172.3 & 25 & 7.57 & 3.75 & 33.8 & 561 & 37.2 & 7.3 & 467 & 1.21 & 40.3 & 489 & 9.8 & 0.491 & 12.4 & 0.231 & 2.63 & 1.75 \\
\hline \multicolumn{19}{|l|}{$122-763 \mathrm{~B}-$} \\
\hline & 5.4 & 35 & & 6.16 & & 56 & 36. & 8.3 & 493 & 0.8 & 40.3 & 49 & 8.9 & 2 & 12.8 & .598 & 1.97 & 0.78 \\
\hline & & 2 & 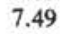 & & & 5 & 34 & 8. & 46 & 2. & 48.9 & 48 & & & 9.0 & 20 & & \\
\hline-150 & 262.4 & 2 & 44 & 7.3 & 32 & 55 & 31.3 & 8. & 493 & 3.31 & 53.3 & 48 & 7.5 & 0.2 & 3.9 & 0.356 & 5.64 & 1.09 \\
\hline $12 X$ & 2 & 3 & & 7.9 & & 556 & 28.5 & 8. & 486 & 85.01 & 51.8 & 48 & & & 3.6 & .414 & 6.34 & 0.56 \\
\hline 15 & 319.4 & 4 & 36 & & & 559 & 26.8 & 9. & 467 & 564.33 & 57.6 & 4 & 7.4 & 0 & 1.4 & 0.383 & 1.83 & 0.47 \\
\hline & 346.4 & 21 & & & & 557 & 25.6 & 10.3 & 411 & & 64.8 & & 7.0 & & 6.1 & 0.335 & 2.69 & 1.42 \\
\hline 24 & 406.4 & 4 & 7.5 & 5. & & 558 & 24.4 & 10.7 & 335 & 302 . & 83.5 & 4 & 6.3 & 0 & 0.7 & 0.764 & 4.46 & 3.29 \\
\hline & & 2 & & 4. & & 557 & 25.2 & ic & 279 & 10.98 & 87.1 & & & & 7.1 & 0.700 & 9.96 & 5.24 \\
\hline-150 & 461. & 2 & 7.3 ? & 4. & & 55 & 24 & & 2. & 41.50 & 102.3 & 48 & 5 & 0 & 1.9 & 0.774 & 9.63 & 5.59 \\
\hline 3 & & 1 & $\cdots$ & 4. & & 556 & 23 & & 271 & 22.79 & 92.2 & 490 & 5.3 & & 2.8 & 0.787 & 1.45 & 6.90 \\
\hline $36 X-4,14$ & 518.9 & 22 & 7.57 & 2.02 & & 55 & 22 & & 278 & 32.03 & 102.3 & 491 & 4.7 & 0.4 & 4.8 & 0.505 & 1.86 & 5.35 \\
\hline 4 & & 5 & & & & 5 & 23 & & 308 & 23.70 & 136.9 & & 3.5 & & 3.4 & 0.374 & & \\
\hline $45 X-3,140-150$ & 6 & 7 & & & & 53 & 24 & & 2 & 9.10 & 145.5 & 48 & 3.2 & & 9.3 & 0.279 & & \\
\hline $50 \times-4,140-150$ & 638.4 & 7 & 7.66 & 3.75 & 30.9 & 537 & 20.0 & 13.9 & 340 & 97.57 & 178.7 & 475 & 3.6 & 0.105 & 3.3 & 0.262 & & \\
\hline \multicolumn{19}{|l|}{$122-763 \mathrm{C}-$} \\
\hline $6 \mathrm{R}-1,140$ & & 7 & .55 & 3.99 & & & 22. & 1 & 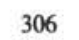 & 13.81 & 10 & 469 & 2.7 & & 3 & 0.181 & & \\
\hline $10 \mathrm{R}-$ & 6 & 10 & & & 31 & 53 & 22 & 14 & 29 & 9.73 & 184.4 & & 3.3 & & 6.2 & 0.187 & 3.87 & 1.51 \\
\hline & & 5 & & & & 539 & & 14 & 30 & 11.17 & 181.5 & & 2.8 & & 3.5 & 0.175 & & \\
\hline $16 \mathrm{R}-5,1$ & 749.5 & 9 & 7.10 & 3.08 & 31 & 540 & 18.2 & 14.7 & 315 & 30.37 & 171.4 & 481 & 2.4 & & 3.6 & 0.152 & 14.5 & 2.28 \\
\hline $19 \mathrm{R}-$ & & 10 & 7.5 & 3. & & 536 & 17.4 & 14.8 & 337 & 228.85 & 158.5 & 477 & 2.2 & & 2.8 & 0.135 & 2.11 & 1.44 \\
\hline $22 \mathrm{R}-4$ & 814.5 & 12 & 7.58 & 3.13 & & 53 & 21.0 & 14.8 & 293 & 21.69 & 134.0 & 485 & 2.6 & 0.1 & 8.9 & 0.137 & 0.63 & 1.07 \\
\hline $25 R$ - & 8 & 2 & & & & 539 & & 15 & 31 & 22.08 & 152.7 & & 2.4 & & 6.2 & 0.162 & & \\
\hline $28 \mathrm{R}-4,1$ & 871. & 7 & 7.34 & 2.95 & 31.7 & 533 & 19.8 & 15.8 & 3? & 8.45 & 136.9 & 472 & 2.2 & 0.245 & 5.3 & 0.187 & & \\
\hline 32 & & 9 & 7.45 & 3.27 & 31 & 532 & 19.8 & 15.1 & 307 & 15.60 & 123.9 & 469 & 2.1 & 0.187 & 3.2 & 0.175 & & \\
\hline $34 \mathrm{R}-5,14$ & 930.0 & 2 & 7.46 & & 31. & 534 & 21.3 & 15.9 & 302 & 16.74 & 158.5 & & 3.0 & & 9.2 & 0.216 & & \\
\hline $41 \mathrm{R}-4,140-150$ & 995.0 & 1 & & & 30.5 & 535 & & & 315 & 24.46 & & & 2.6 & 0.269 & 7.4 & 0.137 & & \\
\hline
\end{tabular}

possibly four $\mathrm{Fe}$ and $\mathrm{Mn}$ maxima were observed at Sites 762 and 763. The Mn maxima overlie those of $\mathrm{Fe}$ as expected on the basis of their respective redox chemistries. However, the maxima at Site 762 are much less pronounced than at Site 763 .

A small dissolved Mn maximum of approximately 2.5 $\mu \mathrm{mol} / \mathrm{L}$ immediately below the sediment surface is followed by a decrease to less than $1 \mu \mathrm{mol} / \mathrm{L}$ between 50 and 100 mbsf. Well-delineated Mn maxima occur near 150-175 mbsf, below which concentrations are again less than $1 \mu \mathrm{mol} / \mathrm{L}$. The dissolved Fe profile at Site 762 is offset slightly deeper within the sediments at Site 762 (near 75 mbsf) relative to Site 763 , but profiles appear to correlate well farther downhole. 
Table 2. Major element composition of Leg 122 sediments.

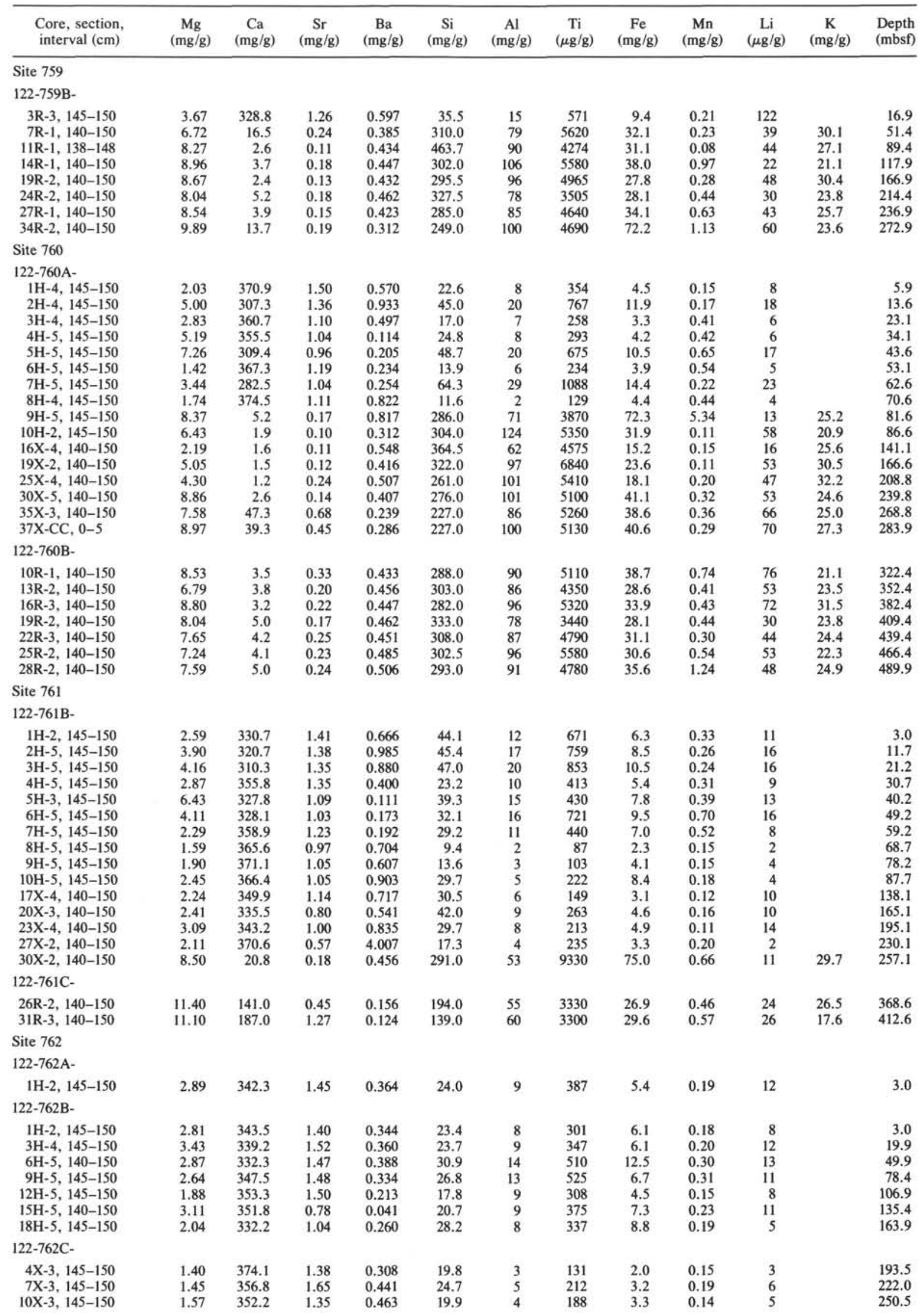


Table 2 (continued).

\begin{tabular}{|c|c|c|c|c|c|c|c|c|c|c|c|c|}
\hline $\begin{array}{l}\text { Core, section, } \\
\text { interval }(\mathrm{cm})\end{array}$ & $\underset{(\mathrm{mg} / \mathrm{g})}{\mathrm{Mg}}$ & $\underset{(\mathrm{mg} / \mathrm{g})}{\mathrm{Ca}}$ & $\underset{(\mathrm{mg} / \mathrm{g})}{\mathrm{Sr}}$ & $\underset{(\mathrm{mg} / \mathrm{g})}{\mathrm{Ba}}$ & $\underset{(\mathrm{mg} / \mathrm{g})}{\mathrm{Si}}$ & $\underset{(\mathrm{mg} / \mathrm{g})}{\mathrm{Al}}$ & $\underset{(\mu \mathrm{g} / \mathrm{g})}{\mathrm{Ti}}$ & $\underset{(\mathrm{mg} / \mathrm{g})}{\mathrm{Fe}}$ & $\underset{(\mathrm{mg} / \mathrm{g})}{\mathrm{Mn}}$ & $\underset{(\mu \mathrm{g} / \mathrm{g})}{\mathrm{Li}}$ & $\underset{(\mathrm{mg} / \mathrm{g})}{\mathrm{K}}$ & $\begin{array}{l}\text { Depth } \\
\text { (mbsf) }\end{array}$ \\
\hline $14 X-5,145-150$ & 2.46 & 331.9 & 1.13 & 0.700 & 36.6 & 7 & 332 & 10.8 & 0.18 & 5 & & 291.5 \\
\hline $17 X-4,145-150$ & 2.18 & 355.5 & 0.92 & 0.730 & 23.6 & 4 & 219 & 8.1 & 0.18 & 2 & & 318.5 \\
\hline $20 \mathrm{X}-2,145-150$ & 2.03 & 361.8 & 0.71 & 1.207 & 22.9 & 5 & 159 & 5.9 & 0.22 & 2 & & 344.0 \\
\hline $23 X-2,145-150$ & 2.45 & 345.1 & 0.78 & 0.969 & 31.3 & 8 & 271 & 7.2 & 0.33 & 7 & & 372.4 \\
\hline $26 \mathrm{X}-4,140-150$ & 1.85 & 327.6 & 0.92 & 0.873 & 48.0 & 7 & 313 & 4.0 & 0.19 & 3 & & 403.9 \\
\hline $29 X-2,140-150$ & 2.65 & 349.0 & 1.13 & 0.404 & 37.3 & 11 & 291 & 10.9 & 0.12 & 9 & & 424.4 \\
\hline $32 X-2,140-150$ & 1.43 & 349.1 & 1.28 & 0.544 & 24.3 & 6 & 231 & 3.0 & 0.09 & 7 & & 452.9 \\
\hline $35 \mathrm{X}-1,140-150$ & 3.24 & 304.3 & 0.99 & 0.569 & 47.0 & 14 & 467 & 7.3 & 0.13 & 14 & & 479.9 \\
\hline $44 X-4,140-150$ & 1.53 & 360.2 & 0.92 & 0.795 & 15.1 & 4 & & 3.1 & 0.18 & 7 & & 569.9 \\
\hline $47 X-5,140-150$ & 1.42 & 358.5 & 1.11 & 0.827 & 14.6 & 5 & 245 & 3.4 & 0.24 & 6 & & 599.9 \\
\hline $50 \mathrm{X}-2,140-150$ & 2.32 & 342.0 & 0.95 & 0.977 & 36.3 & 12 & 577 & 8.2 & 0.31 & 9 & & 623.9 \\
\hline $53 \mathrm{X}-4,140-150$ & 2.88 & 311.6 & 0.86 & 1.701 & 49.7 & 21 & 975 & 15.2 & 0.28 & 26 & & 655.4 \\
\hline $56 \times-5,140-150$ & 2.92 & 322.6 & 0.85 & 3.203 & 46.9 & 17 & 653 & 10.8 & 0.18 & 22 & & 685.4 \\
\hline $59 \mathrm{X}-4,140-150$ & 1.54 & 368.1 & 0.82 & 3.026 & 15.5 & 4 & 211 & 3.7 & 0.11 & 3 & & 712.4 \\
\hline $62 X-4,140-150$ & 1.39 & 388.5 & 0.67 & 0.077 & 10.2 & 2 & 131 & 2.1 & 0.10 & 2 & & 740.9 \\
\hline $66 \mathrm{X}-4,140-145$ & 1.28 & 381.4 & 0.73 & 2.254 & 10.8 & 2 & 106 & 2.2 & 0.13 & 5 & & 770.9 \\
\hline $72 X-1,140-150$ & 3.02 & 331.5 & 0.62 & 9.008 & 36.1 & 11 & 740 & 10.4 & 0.28 & 8 & & 796.4 \\
\hline $77 X-6,140-150$ & 3.70 & 322.3 & 0.29 & 0.837 & 47.3 & 12 & 801 & 7.0 & 0.70 & 10 & & 829.0 \\
\hline $82 X-3,140-150$ & 9.57 & 3.7 & 0.20 & 0.857 & 226.0 & 96 & 6330 & 78.6 & 0.34 & 134 & 34.2 & 857.9 \\
\hline $86 \mathrm{X}-3,140-150$ & 8.23 & 3.4 & 0.14 & 0.818 & 247.6 & 109 & 5888 & 61.3 & 0.31 & 134 & 36.7 & 886.9 \\
\hline $89 X-4,140-150$ & 8.86 & 3.7 & 0.15 & 0.717 & 259.8 & 101 & 5607 & 66.8 & 0.24 & 130 & 31.9 & 916.9 \\
\hline $89 X-4,140-150$ & 8.43 & 3.5 & 0.14 & 0.581 & 251.3 & 94 & 5554 & 65.2 & 0.32 & 130 & 31.4 & 916.9 \\
\hline \multicolumn{13}{|l|}{ Site 763} \\
\hline \multicolumn{13}{|l|}{$122-763 \mathrm{~A}-$} \\
\hline $1 \mathrm{H}-2,145-150$ & 2.74 & 359.4 & 1.51 & 0.426 & 23.1 & 8 & 371 & 5.4 & 0.23 & 7 & & 3.0 \\
\hline $2 \mathrm{H}-4,145-150$ & 2.31 & 347.1 & 1.47 & 0.399 & 27.8 & 7 & 271 & 4.4 & 0.15 & 8 & & 10.9 \\
\hline $3 \mathrm{H}-4,145-150$ & 2.71 & 357.7 & 1.52 & 0.371 & 24.2 & 7 & 300 & 4.6 & 0.17 & 8 & & 20.4 \\
\hline $4 \mathrm{H}-4,145-150$ & 4.65 & 321.4 & 1.37 & 0.379 & 34.6 & 14 & 511 & 8.7 & 0.20 & 15 & & 29.9 \\
\hline $5 \mathrm{H}-4,145-150$ & 2.81 & 354.9 & 1.60 & 0.338 & 21.6 & 9 & 396 & 5.0 & 0.20 & 10 & & 39.4 \\
\hline $6 \mathrm{H}-4,145-150$ & 2.97 & 341.9 & 1.63 & 0.396 & 25.5 & 11 & 403 & 6.4 & 0.24 & 10 & & 48.9 \\
\hline $7 \mathrm{H}-4,145-150$ & 3.05 & 327.2 & 1.52 & 0.415 & 32.0 & 14 & 492 & 8.4 & 0.33 & 10 & & 58.4 \\
\hline $8 \mathrm{H}-4,145-150$ & 3.12 & 337.6 & 1.53 & 0.460 & 33.2 & 16 & 533 & 10.1 & 0.34 & 11 & & 67.9 \\
\hline $9 \mathrm{H}-4,140-150$ & 2.56 & 331.0 & 1.57 & 0.249 & 25.1 & 12 & 447 & 7.3 & 0.26 & 13 & & 77.4 \\
\hline $10 \mathrm{H}-4,140-150$ & 2.30 & 355.4 & 1.01 & 0.967 & 19.0 & 5 & 207 & 4.4 & 0.09 & 4 & & 86.9 \\
\hline $13 \mathrm{H}-4,140-150$ & 1.87 & 360.9 & 1.44 & 0.211 & 14.7 & 6 & 226 & 7.1 & 0.15 & 5 & & 115.4 \\
\hline $16 \mathrm{H}-4,145-150$ & 2.87 & 358.9 & 1.06 & 0.098 & 13.9 & 5 & 208 & 2.9 & 0.29 & 4 & & 143.9 \\
\hline $19 \mathrm{H}-4,145-150$ & 2.00 & 324.2 & 1.06 & 0.321 & 33.5 & 10 & 477 & 8.6 & 0.29 & 6 & & 172.4 \\
\hline \multicolumn{13}{|l|}{$122-763$ B- } \\
\hline $3 \mathrm{X}-4,145-150$ & 1.51 & 352.0 & 1.53 & 0.525 & 24.5 & 6 & 260 & 3.1 & 0.12 & 2 & & 205.5 \\
\hline $6 \mathrm{X}-4,145-150$ & 2.17 & 354.7 & 1.38 & 0.255 & 18.2 & 8 & 308 & 5.0 & 0.22 & 9 & & 234.0 \\
\hline $9 X-4,145-150$ & 3.91 & 316.7 & 0.86 & 2.555 & 49.5 & 17 & 715 & 12.9 & 0.33 & 16 & & 262.5 \\
\hline $12 \mathrm{X}-3,140-150$ & 2.38 & 349.4 & 0.94 & 2.964 & 26.5 & 8 & 347 & 5.6 & 0.06 & 7 & & 289.4 \\
\hline $15 X-4,140-150$ & 3.52 & 376.6 & 0.72 & 2.238 & 14.0 & 3 & 182 & 3.5 & 0.02 & 3 & & 319.4 \\
\hline $18 X-3,140-150$ & 3.67 & 318.0 & 0.58 & 3.920 & 38.4 & 15 & 604 & 9.3 & 0.25 & 16 & & 346.4 \\
\hline $24 \mathrm{X}-5,140-150$ & 4.53 & 199.4 & 0.83 & 2.320 & 132.3 & 28 & 1314 & 16.2 & 1.06 & 15 & & 406.4 \\
\hline $27 \mathrm{X}-4,140-150$ & 4.77 & 115.7 & 0.43 & 0.851 & 239.2 & 25 & 1409 & 47.1 & 1.51 & 12 & & 433.4 \\
\hline $30 \mathrm{X}-4,140-150$ & 9.33 & 137.8 & 0.49 & 2.196 & 198.0 & 39 & 1740 & 21.0 & 2.49 & 22 & & 461.9 \\
\hline $33 \mathrm{X}-5,140-150$ & 6.70 & 149.7 & 0.52 & 1.703 & 179.0 & 32 & 1624 & 15.1 & 0.89 & 17 & & 491.9 \\
\hline $36 \mathrm{X}-4,140-150$ & 5.82 & 208.4 & 0.36 & 1.383 & 120.4 & 29 & 1252 & 20.4 & 1.79 & 15 & & 518.9 \\
\hline $37 \mathrm{X}-1,47-50$ & 8.89 & 180.2 & 0.40 & 1.301 & 174.9 & 40 & 2438 & 26.3 & 0.92 & 26 & & 523.0 \\
\hline $37 \mathrm{X}-2,42-45$ & 7.72 & 198.2 & 0.39 & 1.653 & 162.2 & 36 & 2165 & 21.1 & 0.79 & 22 & 13.5 & 524.4 \\
\hline $37 \mathrm{X}-3,47-50$ & 6.02 & 263.0 & 0.35 & 1.017 & 112.5 & 23 & 1562 & 16.1 & 1.11 & 19 & & 525.9 \\
\hline $37 X-4,47-50$ & 5.47 & 239.9 & 0.37 & 1.335 & 131.3 & 25 & 1554 & 14.2 & 0.92 & 18 & 10.1 & 527.4 \\
\hline $37 \mathrm{X}-5,52-55$ & 9.04 & 149.0 & 0.34 & 1.948 & 198.8 & 47 & 2528 & 24.0 & 0.60 & 38 & 16.4 & 528.9 \\
\hline $38 \times-7,46-49$ & 5.78 & 271.2 & 0.36 & 0.459 & 108.0 & 21 & 1465 & 15.4 & 0.89 & 22 & 7.4 & 532.4 \\
\hline $39 \mathrm{X}-1,47-50$ & 4.05 & 142.0 & 0.20 & 0.357 & 272.2 & 15 & 1101 & 11.2 & 0.43 & 14 & 5.0 & 542.0 \\
\hline $40 X-1,50-53$ & 3.10 & 79.9 & 0.15 & 0.404 & 328.5 & 13 & 1030 & 8.1 & 0.16 & 6 & 4.3 & 551.5 \\
\hline $40 \times-2,50-53$ & 3.74 & 45.8 & 0.12 & 0.593 & 365.2 & 18 & 1525 & 13.2 & 0.16 & 6 & 6.9 & 553.0 \\
\hline $41 X-2,47-49$ & 5.45 & 66.6 & 0.18 & 1.166 & 310.0 & 28 & 2146 & 21.2 & 0.39 & 20 & 10.7 & 561.0 \\
\hline $42 X-2,45-49$ & 10.36 & 3.5 & 0.23 & 2.819 & 325.6 & 58 & 3519 & 41.4 & 0.33 & 48 & 21.9 & 562.5 \\
\hline $42 X-3,44-50$ & 10.49 & 2.3 & 0.23 & 3.210 & 319.3 & 61 & 3407 & 39.7 & 0.29 & 53 & 20.8 & 564.0 \\
\hline $42 X-4,45-49$ & 12.68 & 2.4 & 0.22 & 2.639 & 319.4 & 66 & 3971 & 41.8 & 0.26 & 55 & 20.7 & 565.5 \\
\hline $42 X-5,46-51$ & 9.44 & 3.2 & 0.24 & 3.071 & 317.4 & 56 & 3440 & 39.1 & 0.26 & 39 & 22.1 & 567.0 \\
\hline $42 X-6,47-51$ & 8.56 & 3.9 & 0.24 & 2.897 & 332.9 & 54 & 3358 & 41.4 & 0.54 & 39 & 22.5 & 568.5 \\
\hline $42 X-6,140-150$ & 10.40 & 4.5 & 0.19 & 2.311 & 326.6 & 60 & 3657 & 43.8 & 0.36 & 42 & 22.5 & 578.9 \\
\hline $42 X-74,7-52$ & 9.97 & 3.1 & 0.20 & 2.466 & 318.1 & 56 & 3515 & 43.5 & 0.37 & 44 & 20.9 & 579.4 \\
\hline $43 X-1,44-47$ & 10.38 & 6.3 & 0.27 & 3.620 & 313.2 & 62 & 3482 & 32.7 & 0.28 & 49 & 18.9 & 580.0 \\
\hline $43 X-3,48-50$ & 10.90 & 18.5 & 0.26 & 3.334 & 303.2 & 66 & 3552 & 32.3 & 0.19 & 52 & 20.6 & 583.0 \\
\hline $43 X-4,48-52$ & 10.59 & 2.2 & 0.21 & 2.457 & 320.9 & 62 & 3546 & 38.1 & 0.15 & 50 & 21.3 & 584.5 \\
\hline $45 X-3,140-150$ & 13.54 & 10.2 & 0.24 & 2.746 & 305.3 & 70 & 3030 & 34.1 & 0.13 & 62 & 18.5 & 602.9 \\
\hline $47 X-1,47-49$ & 5.93 & 94.4 & 0.24 & 1.443 & 270.3 & 29 & 1982 & 24.9 & 0.59 & 27 & 11.9 & 618.0 \\
\hline $50 X-4,140-150$ & 7.21 & 3.6 & 0.10 & 0.756 & 269.3 & 86 & 7332 & 62.6 & 0.23 & 98 & 32.4 & 638.4 \\
\hline \multicolumn{13}{|l|}{$122-763 \mathrm{C}-$} \\
\hline $6 \mathrm{R}-1,140-150$ & 6.84 & 2.5 & 0.12 & 0.662 & 290.4 & 90 & 6574 & 49.9 & 0.39 & 91 & 25.5 & 662.0 \\
\hline $10 \mathrm{R}-4,140-150$ & 9.63 & 16.9 & 0.18 & 0.668 & 245.7 & 100 & 5770 & 58.4 & 0.38 & 121 & 29.3 & 691.0 \\
\hline $13 \mathrm{R}-4,140-150$ & 8.46 & 3.1 & 0.11 & 0.705 & 267.9 & 102 & 5912 & 52.8 & 0.44 & 134 & 30.7 & 719.5 \\
\hline
\end{tabular}


Table 2 (continued).

\begin{tabular}{|c|c|c|c|c|c|c|c|c|c|c|c|c|}
\hline $\begin{array}{l}\text { Core, section, } \\
\text { interval }(\mathrm{cm})\end{array}$ & $\underset{(\mathrm{mg} / \mathrm{g})}{\mathrm{Mg}}$ & $\underset{(\mathrm{mg} / \mathrm{g})}{\mathrm{Ca}}$ & $\underset{(\mathrm{mg} / \mathrm{g})}{\mathrm{Sr}}$ & $\underset{(\mathrm{mg} / \mathrm{g})}{\mathrm{Ba}}$ & $\underset{(\mathrm{mg} / \mathrm{g})}{\mathrm{Si}}$ & $\begin{array}{c}\mathrm{Al} \\
(\mathrm{mg} / \mathrm{g})\end{array}$ & $\underset{(\mu \mathrm{g} / \mathrm{g})}{\mathrm{Ti}}$ & $\underset{(\mathrm{mg} / \mathrm{g})}{\mathrm{Fe}}$ & $\underset{(\mathrm{mg} / \mathrm{g})}{\mathrm{Mn}}$ & $\underset{(\mu \mathrm{g} / \mathrm{g})}{\mathrm{Li}}$ & $\underset{(\mathrm{mg} / \mathrm{g})}{\mathrm{K}}$ & $\begin{array}{l}\text { Depth } \\
\text { (mbsf) }\end{array}$ \\
\hline $16 \mathrm{R}-5,140-150$ & 8.45 & 4.9 & 0.11 & 0.673 & 278.1 & 96 & 6145 & 55.1 & 0.17 & 112 & 29.6 & 749.5 \\
\hline $19 R-4,140-150$ & 8.92 & 5.3 & 0.14 & 0.683 & 272.0 & 104 & 6295 & 51.2 & 0.27 & 133 & 31.0 & 776.5 \\
\hline $22 \mathrm{R}-4,140-150$ & 10.14 & 4.1 & 0.13 & 0.664 & 257.2 & 104 & 6085 & 62.7 & 0.35 & 131 & 29.0 & 805.1 \\
\hline $25 \mathrm{R}-5,140-150$ & 10.16 & 3.9 & 0.14 & 0.632 & 254.2 & 108 & 5723 & 60.7 & 0.32 & 144 & 22.4 & 835.1 \\
\hline $28 \mathrm{R}-4,140-150$ & 9.44 & 3.2 & 0.11 & 0.656 & 323.0 & 100 & 6046 & 55.3 & 0.16 & 125 & 27.5 & 862.1 \\
\hline $32 \mathrm{R}-5,140-150$ & 9.01 & 4.6 & 0.10 & 0.618 & 267.6 & 94 & 5499 & 62.0 & 0.30 & 106 & 27.5 & 892.1 \\
\hline $34 \mathrm{R}-5,140-150$ & 10.38 & 4.7 & 0.13 & 0.631 & 257.6 & 107 & 5641 & 62.0 & 0.31 & 127 & 26.9 & 920.6 \\
\hline $37 \mathrm{R}-5,140-150$ & 11.24 & 5.2 & 0.14 & 0.593 & 242.9 & 115 & 5789 & 62.3 & 0.36 & 127 & 25.3 & 949.1 \\
\hline $41 \mathrm{R}-4,140-150$ & 9.72 & 3.1 & 0.12 & 0.648 & 259.1 & 113 & 7926 & 51.8 & 0.25 & 120 & 26.2 & 985.6 \\
\hline
\end{tabular}

\section{DISCUSSION}

\section{Wombat Plateau Sites}

Vertical trends in the pore-water concentrations of $\mathrm{Ca}, \mathrm{K}$, $\mathrm{Mg}, \mathrm{Rb}, \mathrm{Sr}$, and sulfate downhole reflect both diagenesis and the changing bulk sediment chemistry with increasing depth at Sites 759 and 760 . The lack of significant pore-water concentration gradients at Site 761, except for silica, may be attributed largely to the erasure of diffusion profiles by circulation of seawater through predominantly unconsolidated (to weakly lithified) carbonate sediments. Gradients that provide evidence of sedimentary reactions involving silica, however, reflect the extremely reactive nature of this species and its greater dependence on the lithology of the sediments than other pore-water constituents (Gieskes, 1981, 1985).

Because Sites 759 and 760 appear to be more closely related in their sedimentary and pore-water compositions, they are discussed together. Pore-water constituent profiles are rather monotonous at Site 761 and will be discussed principally in terms of silica diagenesis and the occurrence of ferromanganese-rich deposits.

Calcium and $\mathrm{Mg}$ concentration gradients are generally opposite one another and reflect carbonate diagenesis. However, coincident variations below Unit III at Site 760 indicate that carbonate diagenesis does not solely control the concentration of these elements. The increasingly siliciclastic lithology in Units IV through VI (Haq, von Rad, O'Connell, et al., 1990) suggests that ion-exchange reactions in clays also influence the dissolved concentrations of $\mathrm{Ca}$ and $\mathrm{Mg}$. Although a significant portion of the $\mathrm{Mg}$ uptake is attributed to the precipitation of dolomitic limestones, observed in Site 759 and 760 sediments (Haq, von Rad, O'Connell, et al., 1990), uptake of $\mathrm{Mg}$ during the formation of smectite can also be invoked to explain its behavior below Unit III of Site 760 (Kastner and Gieskes, 1976; Gieskes et al., 1981). At Site 761 dissolved $\mathrm{Mg}$ varies by less than $10 \%$ throughout the carbonate oozes and chalks of lithological Units I and II and the ferruginous sandstone of Unit III. It is only in Unit $\mathrm{V}$ that a large depletion of $\mathrm{Mg}$ is observed. Because of the lack of pore-water samples in Unit IV it is not possible to say at which depth the depletion of $\mathrm{Mg}$ becomes significant. Based on the lithology (Haq, von Rad, O'Connell, et al., 1990), Mg depletion may begin in Unit IV where limestones and authigenic clays (Thurow and von Rad, this volume) occur, and continue through the calcareous claystone and crinoidal limestone of Units V and VI. It is likely that extensive diffusion through the unconsolidated oozes and chalks of the upper section of Site 761 has smoothed out concentration gradients (Lasaga and Holland, 1976; Gieskes et al., 1981).

Positive dissolved $\mathrm{Sr}$ gradients creating approximately three-fold (Site 759) to 10-fold (Site 760) enrichments down- hole result primarily from the recrystallization of carbonates. The extent of carbonate recrystallization was evaluated by calculating the $\delta \mathrm{Sr} / \delta \mathrm{Ca}$ ratio within carbonate sediments. Variations in the dissolved concentration of these elements in siliciclastic sediments might result from other reactions, hence no calculation was performed in non-carbonate sediments. Elevated $\delta \mathrm{Sr} / \delta \mathrm{Ca}$ ratios $\left(35-50 \times 10^{-4}\right)$ in the pore water relative to the $\mathrm{Sr} / \mathrm{Ca}$ ratio in nannofossil oozes (approximately $20 \times 10^{-4}$ ) provide evidence of recrystallization (Baker et al., 1982). In Units V through VII, at Site 760, carbonate recrystallization probably continues to increase dissolved $\mathrm{Sr}$ concentrations, but the greater abundance of siliciclastic sediments and ion-exchange reactions in clays may also affect the $\mathrm{Sr}$ abundance. Weaker positive dissolved $\mathrm{Sr}$ gradients within the unconsolidated carbonate sediments of Site 761 suggest a lesser extent of carbonate recrystallization. Overall, the changes in the pore-water concentrations of $\mathrm{Ca}, \mathrm{Mg}$, and $\mathrm{Sr}$ may be attributed to carbonate diagenesis and ion exchange reactions with clay minerals.

Barium does not correlate with the other alkaline earths. Dissolved $\mathrm{Ba}$ concentrations are controlled primarily by the solubility of $\mathrm{BaSO}_{4}$ and are manifested by greater than 10-fold increases downhole. A decrease in concentration toward the seawater value near 214 mbsf at Site 759 is attributed to contamination of the sample with sulfate-enriched drilling water.

Weak sulfate gradients in the upper 80-100 mbsf, which steepen substantially below this depth, suggest limited sulfate reduction associated with the degradation of organic matter within this portion of the sediments, or substantial diffusion. Based on the lithology (Haq, von Rad, O'Connell, et al., 1990) and the amount of pore water squeezed (Table 1) from the recovered sediments (i.e., indicator of the extent of lithification of sediments), the latter explanation appears more plausible. Significantly lower sediment porosity below Units I and II at Sites 759 and 760 (Haq, von Rad, O'Connell, et al., 1990) limits diffusion and helps to maintain steeper sulfate gradients than in the unconsolidated oozes.

Below the oozes, changes in sulfate concentration gradients appear to roughly follow lithological boundaries. A relatively sharp decrease is observed within the $\mathrm{Fe}$ - and Mn-rich sediments of Unit III at Site 760, whereas a similar decrease at Site 759 is only apparent well within lithological Unit III. A minimum (5.2 mM sulfate) in lithological Unit VI (Site 760) is not associated with an alkalinity maximum, suggesting that the bicarbonate produced is consumed by precipitation of calcite (Couture et al., 1977; Gieskes et al., 1987). Sulfate depletion downhole is rather continuous at both sites, indicating that similar processes govern sulfate concentrations throughout the sediments. There is only a weak negative sulfate gradient at Site 761 (Fig. 2 and Table 1). This contrasts sharply with observations at Sites 759 and 760 (80\% depletion near the bottom of the holes) and suggests extensive circulation of seawater throughout Site 761. Alternatively, the 

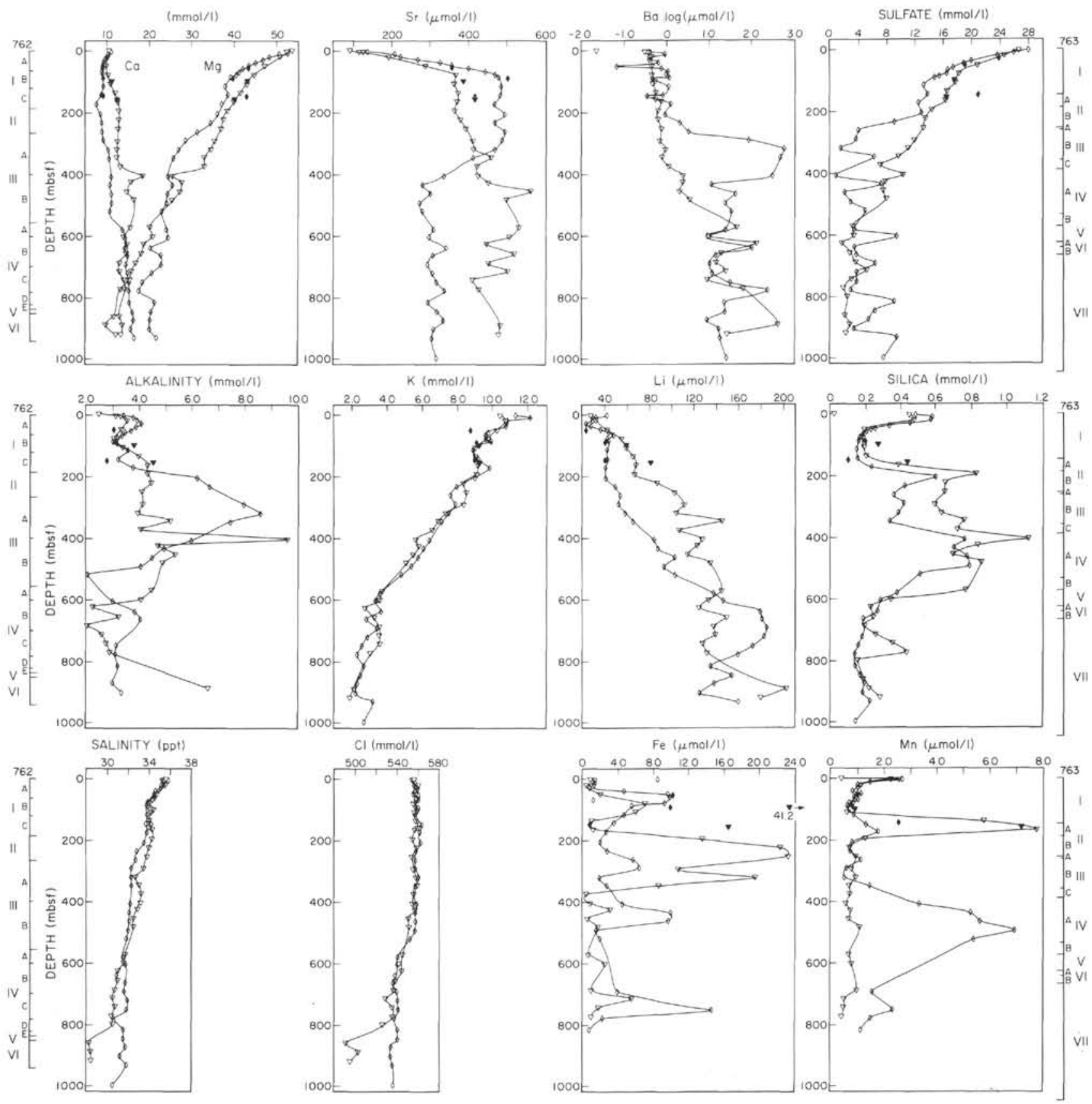

Figure 3. Exmouth Plateau sites: pore-water concentrations as a function of depth with lithological units indicated to the left. Triangles $=$ Site 762, diamonds $=$ Site 763. Closed symbols $=$ "Barnes" harpoon samples; open symbols $=$ squeezed pore-water samples

slight sulfate depletion could be explained by differences in the total organic carbon (TOC) content of the sediments $(<0.10 \%$ above $348 \mathrm{mbsf}$ at Site 761 vs. a range of approximately $1 \%-3 \%$ at Sites 759 and 760 ) (Haq, von Rad, O'Connell, et al., 1990).

The alkalinity profiles at all Wombat Plateau sites are characterized by slight decreases downhole. No significant increases expected as a result of the degradation of organic matter are apparent, although TOC contents of $1 \%-3 \%$ were observed at Sites 759 and 760 . This suggests that any alkalinity produced during sulfate reduction is consumed by precipitation of carbonates. The low TOC content of Site 761 sediments above 348 mbsf is reflected in a nearly constant, although higher, alkalinity than at the previous two sites. This alkalinity increase is compatible with the slight extent of calcite dissolution and sulfate reduction evidenced by higher dissolved $\mathrm{Ca}$ and lower sulfate, respectively.

Except for the uppermost 20 to $30 \mathrm{mbsf}, \mathrm{K}$ and $\mathrm{Mg}$ profiles are similar. Increases in the $\mathrm{K}$ concentration within lithologic Unit I at all sites are likely due to temperature of squeezing effects (Bischoff et al., 1970). The onset of a negative $\mathrm{K}$ gradient below 400 mbsf at Site 761 coincides with a change in the lithology from predominantly marine carbonate-rich sediments (Subunit VA) to a greater proportion of clay-rich delta plain sediments (Subunits VB-VI) (e.g., Table 2; Haq, von Rad, O'Connell, et al., 1990; Wilkens et al., this volume). 
The dissolved Li profiles at Sites 759 and 760 are influenced by diagenetic processes as well as by the presence of clays. There is a weak relationship between the dissolved $\mathrm{Li}$ and silica concentrations throughout most of the sediments. Within the carbonate oozes (Units I and II) Li concentrations may reflect its release during the dissolution of amorphous silica. Deeper downhole, however, the variations in dissolved $\mathrm{Li}$ correlate with changes in the lithology from carbonates to claystones, and likely result from uptake of $\mathrm{Li}$ by clay minerals. It should be noted that $\mathrm{Li}$ and silica profiles are completely decoupled at Site 761 .

The large variations in dissolved silica throughout the Wombat Plateau sediments are likely due to silica diagenesis although no simple inverse relationship between silica and $\mathrm{Ca}$ or alkalinity, which would reflect silica diagenesis (Gieskes, 1981), exists in the sediments at Sites 759 or 760 . Rather a relationship between $\mathrm{Mg}$ and $\mathrm{SiO}_{2}$ at Site 760 is consistent with the uptake of $\mathrm{Mg}$ and $\mathrm{SiO}_{2}$ during the formation of smectite (Kastner and Gieskes, 1976); however, the various fields in Figure 4 suggest that dissolved concentrations of these two species are influenced by different reactions in the various lithological units.

Much greater variability is noted in the dissolved silica concentrations at Site 761 (Table 1). A strong negative gradient within lithological Unit I coincides with the disappearance of radiolaria from the sediments (Schrader, 1972). From approximately 50 to $140 \mathrm{mbsf}$ the strong positive gradient $(0.643 \mathrm{mmol} / \mathrm{L} / 100 \mathrm{~m})$, with a maximum of $0.78 \mathrm{mmol} / \mathrm{L}$ at 138 mbsf, probably results from the dissolution of skeletal opal-A. It correlates with the transition from lithological Subunits IB to IC in which massive cherts exist (Haq, von Rad, O'Connell, et al., 1990) and reflects major $\mathrm{SiO}_{2}$ mobilization and precipitation of authigenic silica. The gradual decrease to near 0.37 $\mathrm{mmol} / \mathrm{L}$ at $257 \mathrm{mbsf}$ and further to $0.186 \mathrm{mmol} / \mathrm{L}$ downhole is inferred to represent incorporation of silica into clay minerals (Kastner and Gieskes, 1976) or, perhaps, equilibrium with opal-CT. It coincides with the presence of dark laminated claystone in Subunit VB.

Decreases in pore-water salinity from the seawater value to approximately $32 \mathrm{~g} / \mathrm{kg}$ can be largely attributed to the loss of $\mathrm{K}, \mathrm{Mg}$, and sulfate with increasing depth downhole. Salinity and dissolved $\mathrm{Cl}$ at Site 760 decrease most $(35.5-32.6 \mathrm{~g} / \mathrm{kg}$ and $568-521 \mathrm{mmol} / \mathrm{L}$, respectively) between 82 and $86 \mathrm{mbsf}$ near the boundary between lithological Unit III, a Mn oxiderich sandstone and siltstone interval, and Unit IV sediments comprised of claystones with root-mottling and minor coal. The sympathetic $\mathrm{Cl}$ and salinity changes throughout Units III and IV suggest a freshwater intrusion near the top of Unit IV. However, Gieskes (1974) has indicated that an input of freshwater should only be considered if the salinity drops below $31.5 \mathrm{~g} / \mathrm{kg}$. At Site 760 there is no correlation between sediment porosity and $\mathrm{Cl}$ or salinity. The porosity at the boundary of Units II and III is near 70\%, it is near $60 \%$ at 82 mbsf, and approximately $50 \%$ at 86 mbsf and decreases further throughout Unit IV (Haq, von Rad, O'Connell, et al., 1990). Greater freshwater flow would be expected through the upper section of Unit III than near the boundary of Units III and IV. Chloride increases below Unit IV, whereas a loss of $\mathrm{K}, \mathrm{Mg}$, and sulfate leads to a salinity decrease. Hence, the less saline waters at 86 mbsf are a localized occurrence within the sedimentary column that may result from horizontal advection of fresh waters from the Australian continent. Similar occurrences of lateral transport of fluids (both more and less saline than seawater) at depth have been reported previously and are generally associated with sands or siltstones (Couture et al., 1977; Gieskes, 1981).

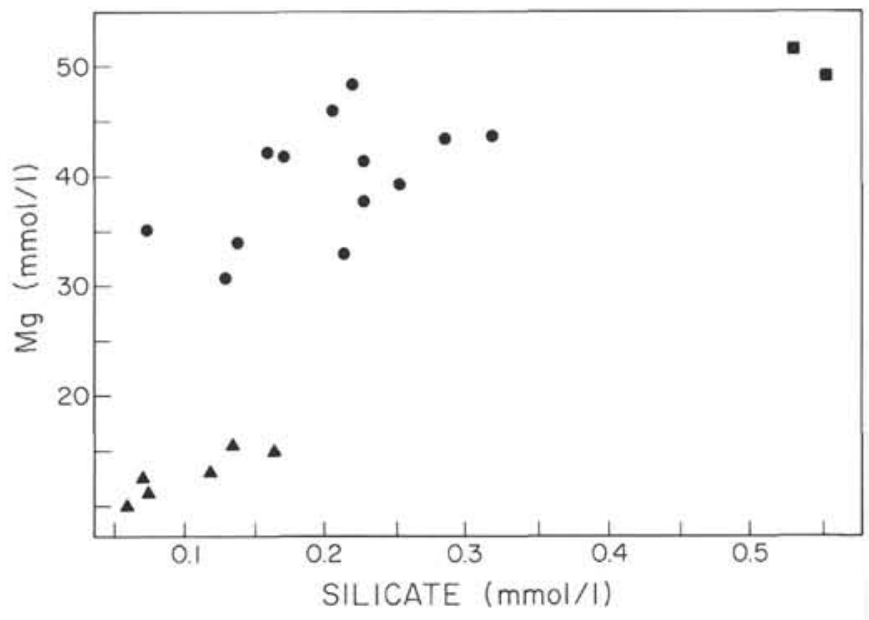

Figure 4. $\mathrm{Mg}$ vs. $\mathrm{SiO}_{2}$ at Site 760 . Squares $=$ Unit $\mathrm{I}$, circles $=$ Units II-V, and triangles = Units VI and VII.

Dissolved $\mathrm{Fe}$ and $\mathrm{Mn}$ profiles appear to reflect changes in redox conditions within the first 50 mbsf of the sediments (Fig. 2 and Table 1). Elevated Mn concentrations immediately below the sediment/seawater interface and higher Fe concentrations from about 5-20 mbsf follow the conventional redox sequence associated with the degradation of organic matter in sediments. A large dissolved $\mathrm{Mn}$ maximum ( $39 \mu \mathrm{mol} / \mathrm{L}$ ) at $81.6 \mathrm{mbsf}$ (Site 760 ) coincides with the occurrence of ferromanganese-rich sediments and crusts in lithological Unit III (e.g., De Carlo and Exon, this volume). A smaller, yet well-defined, maximum is also observed at 50 mbsf in the sediments of Site 761, although no Fe-Mn-rich sediments were found that could explain the higher dissolved Mn concentrations. Pore waters from the Wombat Plateau sites generally exhibit an increase in dissolved $\mathrm{Fe}$ immediately below the Mn maxima, although the former is quite small at Site 761, and no downhole Mn maximum is observed at Site 759. The local maximum of approximately $9 \mu \mathrm{mol} / \mathrm{L} \mathrm{Fe}$ associated with a pH of 8.65 at 141 mbsf in Unit IV of Site 760 is unusual, particularly considering the high $\mathrm{pH}$, and the reason for this remains unclear. The low pore-water $\mathrm{Fe}$ and much higher Mn concentrations in Unit III suggest that redox conditions are suboxic within this interval, but are not sufficiently reducing to cause dissolution of $\mathrm{Fe}$ oxides. The dissolved $\mathrm{Fe}$ and $\mathrm{Mn}$ profiles of Site 761 suggest that variable redox conditions exist over the first 80 mbsf. Although no obvious Fe-Mn-rich intervals were noted in the core descriptions, white, brown, and gray layers occur over $10-$ to $30-\mathrm{cm}$ intervals and extend to the lower boundary of Subunit IA in Core 122-761B-8H. Colored layers were also observed in Subunit IB to 90.5 mbsf (Haq, von Rad, O'Connell, et al., 1990), below which the concentrations of these metals return to background levels. The color banding may reflect variable redox conditions although shipboard descriptions suggest that the color changes are associated with variations in the ratio of foraminifers to nannofossils in the sediments.

\section{Sites 762 and 763}

The strong negative $\mathrm{Mg}$ gradient is not as pronounced in the upper $100 \mathrm{~m}$ of Site 762 as in the corresponding section of Site 763. However it is, on average, almost linear down to approximately 800 and $500 \mathrm{mbsf}$ at the two sites, respectively. These depths correspond roughly to the limit below which the sediments are more lithified or grade from predominantly carbonates to a greater abundance of siliciclastic matter (Haq, von Rad, O'Connell, et al., 1990). Much smaller Mg gradients exist below 
these depths, suggesting that the Mg diffusion profile is strongly affected by the extent of carbonate sedimentation. The existence of at least three changes in slope in the dissolved $\mathrm{Mg}$ profile in the carbonate sequence of Site 763 reflects changes in the lithology, hence, porosity of the sediments, that are more pronounced than at Site 762. Concentration reversals noted in the lower $400 \mathrm{~m}$ at Site 763 , where the sediments grade from predominantly calcareous to siliceous-rich material interspersed with dolomitic limestone nodules and beds, suggest that both dolomitization and uptake by clay minerals affect the dissolved $\mathrm{Mg}$ profile in this interval.

Unlike $\mathrm{Mg}$, pore-water $\mathrm{Ca}$ displays only slight changes with depth. An initial small decrease observed at both sites is consistent with calcite precipitation as proposed to occur in the sediments of Site 765 (Compton, in press). Slight increases in dissolved $\mathrm{Ca}$ characterize the rest of the sedimentary sequence at Site 763, whereas a weak negative Ca gradient is observed below $400 \mathrm{mbsf}$ at Site 762 . The relatively large increase in the $\mathrm{Ca}$ concentration at $404 \mathrm{mbsf}$ (Site 762) is accompanied by a two-fold increase in alkalinity and sharp $\mathrm{Mg}$ depletion, and suggests that calcite is dissolving in this inter$\mathrm{val}$ in addition to reactions that remove $\mathrm{Mg}$ from solution. Increases in pore-water $\mathrm{Ca}$ at Site 763 between 579 and 930 mbsf, in the sediments of Units V-VII, are primarily associated with terrigenous silica-rich sediments and reactions involving clay minerals.

The $\mathrm{Sr}$ profiles mainly reflect the recrystallization of calcite. Extremely strong positive gradients over the first 100 mbsf at both sites are followed by a more subtle increase down to near 400 mbsf at Site 762, whereas a zone of near constant $\mathrm{Sr}$ concentration exists between 100 and 350 mbsf at Site 763 . The high $\delta \mathrm{Sr} / \delta \mathrm{Ca}$ ratios $\left(\sim 100 \times 10^{-4}\right)$ in the carbonates of both sites are indicative of calcite recrystallization (Baker et al., 1982). Dissolved Sr variations below 424 mbsf within lithological Subunit IIIB (Site 762) are likely related to slight changes in lithology in addition to calcite recrystallization.

At Site 763 the zone of recrystallization is well-delineated by a narrow concentration range of $467-500 \mu \mathrm{mol} / \mathrm{L} \mathrm{Sr}$ between 90 and $320 \mathrm{mbsf}$. However, $\mathrm{Sr}$ decreases to near 300 $\mu \mathrm{mol} / \mathrm{L}$ and remains less than $400 \mu \mathrm{mol} / \mathrm{L}$ in lithological Unit IV in which nannofossil claystones, claystones, and zeolites are first found. Mirror image behavior between $\mathrm{Sr}$ and $\mathrm{Mg}$ from 600 mbsf to total depth is likely associated with limestone nodule beds (recrystallized calcite and dolomite) within this interval, and suggests that $\mathrm{Sr}$ release may accompany $\mathrm{Mg}$ uptake during dolomitization.

As in other Leg 122 sites, dissolved $\mathrm{Ba}$ is governed by the solubility of $\mathrm{BaSO}_{4}$. Concentrations are generally less than 1 $\mu \mathrm{mol} / \mathrm{L}$ unless sulfate concentrations decrease below 7 $\mathrm{mmol} / \mathrm{L}$. The first of several large concentration fluctuations at Site 762 occurs at 404 mbsf, where most other constituents also exhibit sharp deviations from their normal trends. Subsequent pore-water $\mathrm{Ba}$ increases either coincide with local sulfate minima, or occur where its concentration is below approximately $6 \mathrm{mmol} / \mathrm{L}$. Large crystals of barite were found within the chalks of Subunit IIIB at Site 762 (e.g., Sample 122-762C-42X-6, 110-112 cm) (Haq, von Rad, O'Connell, et al., 1990), attesting to the occurrence of precipitation and dissolution reactions within the sediments. The first of three elevated dissolved $\mathrm{Ba}$ concentrations at Site 763 coincides with the alkalinity maximum and sulfate minimum at 319 mbsf. A strong smell of $\mathrm{H}_{2} \mathrm{~S}$ in cores recovered between 300 and 400 mbsf (Haq, von Rad, O'Connell, et al., 1990) indicates anoxic reducing conditions; the sulfate depletion allows more $\mathrm{Ba}$ to dissolve. The abundance of pyrite and centimeter-scale marcasite nodules further attests to the anoxic nature of these sediments. Below 400 mbsf dissolved $\mathrm{Ba}$ concentrations de- crease but remain significantly higher than observed in sulfaterich pore water. Pyrite and marcasite also become much rarer below 500 mbsf. Two other Ba maxima farther downhole are not as well delineated nor accompanied by high alkalinities. They do, however, occur in sediments where pyrite is common, thereby indicating the presence of sulfide.

The pore-water $\mathrm{Ba}$ spikes were originally discovered when analyzing the glass vial splits (Table 2). Reanalysis of plastic stored splits revealed similar trends, although significantly lower concentrations were found. A comparison of $\mathrm{Ba}$ data from the glass and plastic splits is presented in Table 3 . Because considerably fewer alkalinity splits were available for reanalysis, the

Table 3. Ba stored in glass vs. plastic.

\begin{tabular}{|c|c|c|c|}
\hline $\begin{array}{l}\text { Core, section, } \\
\text { interval }(\mathrm{cm})\end{array}$ & $\begin{array}{l}\text { Depth } \\
\text { (mbsf) }\end{array}$ & $\begin{array}{c}\mathrm{Ba}^{\mathrm{a}} \\
(\mathrm{mg} / \mathrm{L})\end{array}$ & $\begin{array}{c}\mathrm{Ba}^{\mathrm{b}} \\
(\mathrm{mg} / \mathrm{L})\end{array}$ \\
\hline \multicolumn{4}{|l|}{ 122-759B- } \\
\hline $3 R-3,145-150$ & 16.9 & 0.086 & 0.061 \\
\hline $7 \mathrm{R}-1,140-150$ & 51.4 & 0.152 & 0.065 \\
\hline $11 \mathrm{R}-1,138-148$ & 89.4 & 0.204 & 0.125 \\
\hline $14 \mathrm{R}-1,140-150$ & 117.9 & 0.308 & 0.215 \\
\hline \multicolumn{4}{|l|}{$122-760 \mathrm{~B}-$} \\
\hline $13 R-2,140-150$ & 352.4 & 0.240 & 0.194 \\
\hline $16 \mathrm{R}-3,140-150$ & 382.4 & 0.320 & 0.317 \\
\hline $19 \mathrm{R}-2,140-150$ & 409.4 & & 0.329 \\
\hline $22 \mathrm{R}-3,140-150$ & 439.4 & 0.405 & 0.393 \\
\hline \multicolumn{4}{|l|}{$122-761 B-$} \\
\hline $1 \mathrm{H}-2,145-150$ & 2.9 & 0.060 & 0.058 \\
\hline $2 \mathrm{H}-5,145-150$ & 11.6 & 0.075 & 0.049 \\
\hline $3 \mathrm{H}-5,145-150$ & 21.1 & 0.060 & 0.043 \\
\hline $4 \mathrm{H}-5,145-150$ & 30.6 & 0.069 & 0.039 \\
\hline $5 \mathrm{H}-5,145-150$ & 40.1 & 0.065 & 0.059 \\
\hline $6 \mathrm{H}-5,145-150$ & 49.8 & 0.050 & 0.027 \\
\hline 7H-5, 145-150 & 59.1 & 0.051 & 0.034 \\
\hline $8 \mathrm{H}-5,145-150$ & 68.6 & 0.050 & 0.044 \\
\hline $9 \mathrm{H}-5,145-150$ & 78.1 & 0.053 & 0.039 \\
\hline $10 \mathrm{H}-5,145-150$ & 87.6 & 0.063 & 0.048 \\
\hline $17 X-4,140-150$ & 138.1 & 0.075 & 0.063 \\
\hline $23 \mathrm{X}-4,140-150$ & 195.1 & 0.080 & 0.049 \\
\hline $30 X-2,140-150$ & 257.1 & 0.045 & 0.043 \\
\hline \multicolumn{4}{|l|}{$122-762 C$ - } \\
\hline $4 X-3,145-150$ & 193.4 & 0.618 & 0.567 \\
\hline $10 X-4,145-150$ & 251.9 & 0.742 & 0.699 \\
\hline $14 X-5,145-150$ & 291.4 & 0.655 & 0.699 \\
\hline $17 X-4,145-150$ & 318.4 & 0.910 & 0.757 \\
\hline $20 X-2,145-150$ & 343.9 & 0.764 & 0.779 \\
\hline $23 \mathrm{X}-2,140-150$ & 372.4 & 1.092 & 0.975 \\
\hline $26 \mathrm{X}-4,140-150$ & 403.9 & 2.381 & 2.228 \\
\hline $32 X-2,140-150$ & 452.9 & 1.973 & 2.148 \\
\hline $35 X-1,140-150$ & 479.9 & 3.371 & 3.289 \\
\hline $44 X-4,140-150$ & 569.9 & 42.23 & 9.39 \\
\hline $50 X-2,140-150$ & 623.9 & 124.5 & 9.17 \\
\hline $53 X-4,140-150$ & 655.4 & 18.8 & 11.8 \\
\hline $59 X-4,140-150$ & 712.4 & 22.6 & 15.1 \\
\hline $62 X-4,140-150$ & 740.9 & 8.81 & 6.50 \\
\hline $66 X-4,140-150$ & 770.9 & 60.2 & 35.4 \\
\hline $86 X-3,140-150$ & 886.9 & 392 & 30.9 \\
\hline \multicolumn{4}{|l|}{$122-763 B-$} \\
\hline $3 X-4,145-150$ & 205.4 & 0.890 & 0.910 \\
\hline $9 X-4,145-150$ & 262.4 & 3.31 & 3.39 \\
\hline $12 X-3,140-150$ & 289.4 & 85.0 & 11.1 \\
\hline $15 X-4,140-150$ & 319.4 & 564 & 215 \\
\hline $18 X-3,140-150$ & 346.4 & 468 & 66.4 \\
\hline $24 X-5,140-150$ & 406.4 & 303 & 13.7 \\
\hline $27 \mathrm{X}-4,140-150$ & 433.4 & 11.0 & 7.35 \\
\hline $30 X-4,140-150$ & 461.9 & 41.5 & 21.5 \\
\hline $33 X-5,140-150$ & 491.9 & 22.8 & 22.3 \\
\hline $36 \times-4,140-150$ & 518.9 & 32.0 & 35.7 \\
\hline
\end{tabular}

${ }^{a}$ Glass vial splits.

${ }^{b}$ Alkalinity splits stored in plastic. 
glass vial data have been used in Figure 3 to delineate trends. These are generally similar but several pore-water $\mathrm{Ba}$ maxima observed at Site 762 are not as evident from the alkalinity split analyses. Differences between the two splits are generally greater in samples displaying high Ba concentrations; the reasons for these discrepancies remain unclear.

The profiles shown in Figure 3 reveal extensive sulfate reduction associated with the degradation of organic matter at both Exmouth Plateau sites. Sulfate is nearly $75 \%$ depleted within the first 370 mbsf at Site 762 . Downhole sulfate concentrations remain low, except for the local maximum of $10.2 \mathrm{mmol} / \mathrm{L}$ at $404 \mathrm{mbsf}$. The sulfate profile suggests that a reaction zone exists below approximately $600 \mathrm{mbsf}$. At Site 763 sulfate is depleted by more than $50 \%$ within the first $150 \mathrm{~m}$ of the sediment. A change in slope in the sulfate gradient near 150 mbsf that coincides with lithologic Unit IIA (Haq, von Rad, O'Connell, et al., 1990) likely reflects changes in the diffusion coefficient of the sediments. Based on the sulfate minimum of $1.4 \mathrm{mmol} / \mathrm{L}$ at $319 \mathrm{mbsf}$ that corresponds to the alkalinity, $\mathrm{Ba}$, and $\mathrm{Ca}$ maxima mentioned above, it is likely that a reaction zone exists near 320 mbsf, a much shallower depth than at Site 762. The variable sulfate profiles in the deeper sections of each site are not a result of analytical error; they likely result from core contamination with seawater because the concentration changes roughly correlate with dissolved $\mathrm{Cl}$ and $\mathrm{Mg}$. Overall, sulfate concentrations at both sites are suggestive of diffusion profiles affected by variations in the porosity of the sediments, and suggest that reaction zones exist near the transition from chalks to claystones.

The single-point alkalinity maximum at 404 mbsf at Site 762 that has been previously noted to correspond to significant excursions in the concentrations of many other constituents suggests a zone of calcite dissolution. Above and below this depth alkalinity remains in a range of $2-5 \mathrm{mmol} / \mathrm{L}$. The alkalinity of Site 763 pore water, which is characterized by a broad, well-defined maximum at $320 \mathrm{mbsf}$, has been discussed in terms of its association with $\mathrm{H}_{2} \mathrm{~S}$ and sulfate minima, and strongly influences the solubilities of $\mathrm{Ba}, \mathrm{Fe}$, and $\mathrm{Mn}$.

The depletion of dissolved $\mathrm{K}$ down to approximately 600 mbsf generally correlates with $\mathrm{Mg}$ although the two $\mathrm{K}$ profiles are more similar than the corresponding $\mathrm{Mg}$ profiles. This suggests that different reactions may influence the concentrations of $\mathrm{K}$ and $\mathrm{Mg}$ in the upper sections of both sites. Below 600 mbsf pore-water $\mathrm{K}$ decreases farther within the siliciclastic sediments of both sites and correlates better with dissolved $\mathrm{Mg}$, presumably as a result of ion-exchange reactions with clay minerals. As noted at the Wombat sites, slight dissolved $\mathrm{K}$ enrichments over seawater within the first $50 \mathrm{mbsf}$ can most likely be attributed to temperature of squeezing effects (Bischoff et al., 1970).

Dissolved $\mathrm{Li}$ concentrations correlate roughly with $\mathrm{Sr}$ between 340 and 800 mbsf at Site 762. Gieskes et al. (1983) have reported an association of these elements at Deep Sea Drilling Project (DSDP) Sites 474, 482, and 485, which they attributed to the diagenesis of opaline silica and the associated alteration of volcanic materials. A broad silica maximum that correlates roughly with the $\mathrm{Li}$ profile at Site 762 is in agreement with the observations of Gieskes et al. (1983); however, it is unlikely that the alteration of volcanic matter is significant at Site 762 because of the predominance of carbonate and terrigenous sediments in the upper and lower portions of this site, respectively. At Site 763, a notable increase in dissolved $\mathrm{Li}$ occurs in the semilithified chalks of Unit II through Unit V. The well-defined $\mathrm{Li}$ maximum between 638 and $720 \mathrm{mbsf}$ (in Unit VI and the top of Unit VII) likely represents a reaction zone. Below this depth changes in the dissolved $\mathrm{Li}$ concentrations can also be related to silica diagenesis as at many
DSDP sites (e.g., Gieskes et al., 1983). The zone of maximum Li concentration, immediately below that where silica concentrations decrease abruptly at Site 763 , provides evidence in support of this hypothesis, and may be associated with the conversion of cherts to quartz.

The dissolved silica profiles are remarkably similar throughout Sites 762 and 763 although lithological boundaries between carbonates and siliclastic sediments occur at different depths at each site (Haq, von Rad, O'Connell, et al., 1990).

Because of the large Maestrichtian-Eocene hiatus at Site 763 sediment compositions are substantially different at the two sites below 570 mbsf. More carbonate-rich sediments occur at the former and more siliciclastic/terrigenous sediments abound at the latter. Nonetheless, dissolved silica profiles correlate well with the sedimentary composition. Somewhat elevated concentrations (near $0.47 \mathrm{mmol} / \mathrm{L}$ ) near the sediment surface coincide with the onset of radiolarian dissolution (e.g., Blome, this volume). Within the first $50 \mathrm{mbsf}$ of both sites radiolarians disappear and dissolved silica decreases to near $0.2 \mathrm{mmol} / \mathrm{L}$. It remains in this range to $150 \mathrm{mbsf}$ and subsequently rises sharply to $0.4-0.8 \mathrm{mmol} / \mathrm{L}$ at $200 \mathrm{mbsf}$. Fluctuations in dissolved silica down to 570 mbsf (Site 762), including a large local maximum $(\sim 1.2 \mathrm{mmol} / \mathrm{L})$ at $404 \mathrm{mbsf}$ are likely related to the conversion of amorphous silica to opal-CT. In the lower portions of both sites (600 mbsf total depth), concentrations generally well below 0.5 $\mathrm{mmol} / \mathrm{L}$ suggest uptake of silica during the formation of quartz, although equilibrium with quartz should lead to much lower concentrations near $0.1 \mathrm{mmol} / \mathrm{L}$. The effect of different lithologies at the two sites appears as a slight upward displacement of the zone in which dissolved silica concentrations drop to less than $0.5 \mathrm{mmol} / \mathrm{L}$ at Site 763 .

Similarities also exist with the dissolved silica profile at Site 761 although the zone of high reactivity, defined by concentrations between $0.4-1 \mathrm{mmol} / \mathrm{L}$, is compressed over a $200-\mathrm{m}$ interval in the latter. These observations indicate that silica diagenesis is not only dependent on burial depth but is also influenced by both the age and the local lithology of the sediment, in agreement with the findings of Reich and von Rad (1979).

Pore-water salinity decreases at Sites 762 and 763 reflect the depletion of $\mathrm{K}, \mathrm{Mg}$, and sulfate down to approximately 500 mbsf. This is substantiated by the relatively constant $\mathrm{Cl}$ profiles (Fig. 3) to this depth. Slight decreases in $\mathrm{Cl}$ subsequently observed at both sites contribute to the further decrease in salinity down to 800 mbsf. The sharp decrease in salinity and $\mathrm{Cl}$ at 850 mbsf at Site 762 occurs at the transition between the calcareous claystones of lithological Unit V and the terrigenous claystones of Unit VI (Haq, von Rad, O'Connell, et al., 1990). At Site 763 the less-pronounced dissolved Cl decrease also coincides with lithological Unit V and the onset of predominantly terrigenous sediments.

Sharp decreases in salinity and $\mathrm{Cl}$ can be explained by either the decomposition of gas hydrates or by a lateral input of freshwater (Harrison et al., 1982; Gieskes, 1981; Jenden and Gieskes, 1983). The former can be ruled out because no clathrates were found. It is interesting to note that the low-salinity water $(\sim 28 \mathrm{~g} / \mathrm{kg}$, Site 762$)$ occurs within the silty-claystone sediments of the Barrow Group equivalent. Because of the predominantly terrigenous nature of these sediments, which extend to the continent, advection of freshwater from Australia is inferred.

Gieskes (1981) proposed that salinities below $31.5 \mathrm{~g} / \mathrm{kg}$ can be explained by infusion of freshwater at depth in the sediments. Data herein support this hypothesis not only because of the $\mathrm{Cl}$ decrease associated with the salinity change, but because the low salinity and $\mathrm{Cl}$ zones at both sites correlate (lithologically). Limited freshwater infusion at Site 763 compared to Site 762 may 
result from the lower porosity at the former. Porosities decrease from $40 \%$ at $600 \mathrm{mbsf}$ to $20 \%$ at the bottom of Site 763 , in the lithologically equivalent interval at Site 762 they range from $40 \%-50 \%$, whereas the sediments immediately above the low salinity interval have less than $25 \%$ porosity (Haq, von Rad, O'Connell, et al., 1990). A low salinity ( $24 \mathrm{~g} / \mathrm{kg}$ at $801 \mathrm{mbsf}$ ) was also observed near a lithologic boundary in the sediments at Site 765 (Ludden, Gradstein, et al., 1990). At DSDP Site 241 Gieskes (1974) reported the presence of freshwater at least $300 \mathrm{~km}$ from shore; similar observations were made at Site 270 in the Ross Sea (Mann and Gieskes, 1975) and at Site 339 on the Vфring Plateau (Gieskes et al., 1978). In most cases the sediments consisted of permeable sandstones that allow substantial flow (Gieskes, 1981). The sediments of Unit VI at Site 762 are nearly twice as permeable as those immediately above, thus restricting diffusion of freshwater into overlying claystones of Unit V.

Although both dissolved $\mathrm{Fe}$ and $\mathrm{Mn}$ were determined in the surface water samples, these are likely contaminated because they were collected from the ship while on station. Indeed, the measured Mn contents $(0.44$ and $0.47 \mu \mathrm{mol} / \mathrm{L})$ are two to three orders of magnitude higher than normal open-ocean water $(0.2-3$ $\mathrm{nmol} / \mathrm{L}$ ) and at least one order of magnitude greater than observed in continental shelf surface water (Bruland, 1983). The measured concentration of $\mathrm{Fe}(70 \mathrm{nmol} / \mathrm{L})$ in surface water from Site 763 is also at least an order of magnitude greater than would be expected in open-ocean water; furthermore, $\mathrm{Fe}$ concentrations in continental shelf waters are normally extremely low due to its rapid oxidation and removal from seawater (Emerson et al., 1979). Contamination of Fe, possibly from the sampler itself, is also evident in two samples collected with the Barnes "harpoon" (e.g., Table 1, Samples 122-762B-12X-1, 0-10 cm, and 122-762B-18X-1, 0-10 cm), whereas Mn data appear consistent with those from the nearest "squeezed" porewater sample. Because all samples were squeezed using the stainless steel press and no laminar flow hoods were available for "clean sample handling techniques," all Fe concentrations less than 0.5 $\mu \mathrm{mol} / \mathrm{L}$ are potentially suspect. Contamination is not as problematic for Mn and a lower limit of reliability is likely for this element.

Low Mn concentrations at Site 763 throughout the range of the alkalinity maximum combined with strong $\mathrm{H}_{2} \mathrm{~S}$ odors indicate that precipitation of highly insoluble $\mathrm{MnS}$ is likely the cause of low dissolved Mn within this interval. This is supported by the large well-defined Mn maximum immediately below this region and which peaks at 492 mbsf. Pore-water Fe profiles are more scattered and exhibit a wider concentration range than those of $\mathrm{Mn}$. A local minimum also occurs at 319 mbsf below which $\mathrm{Fe}$ increases to near $10 \mu \mathrm{mol} / \mathrm{L}$ in the interval where high $\mathrm{Mn}$ is observed. Another peak in Fe between 700 and 750 mbsf at both sites that is accompanied by a small dissolved Mn increase only at Site 763. Although not well delineated, because of the paucity of pore-water data in the overlying sediments, these correspond to an interval characterized by both pyritized burrows and abundant siderite zones and/or concretions (Haq, von Rad, O'Connell, et al., 1990).

\section{SUMMARY AND CONCLUSIONS}

Similarities in pore-water profiles are observed throughout the sediments cored during Leg 122. Paramount among these are large depletions of $\mathrm{K}, \mathrm{Mg}$, and sulfate. Another similarity is the generally slight variance of $\mathrm{Ca}$ and alkalinity with depth. Within the unconsolidated oozes of all sites, sulfate reduction associated with the bacterial degradation of organic matter should lead to substantial increases in alkalinity. Although local increases are observed at Sites 762 and 763 a slight decrease or no change in alkalinity downhole is more commonly observed. The generally low alkalinities, as well as the absence of large positive $\mathrm{Ca}$ gradients that would be expected from the exchange of $\mathrm{Mg}$ for $\mathrm{Ca}$ in carbonates, indicate that $\mathrm{Ca}$ and alkalinity are consumed by reprecipitation of calcite.

Calcite recrystallization in Leg 122 sediments is evidenced by the high pore-water $\delta \mathrm{Sr} / \delta \mathrm{Ca}$ ratios observed at all sites. The $\delta \mathrm{Sr} / \delta \mathrm{Ca}$ ratio of Leg 122 carbonates ranges from approximately $35 \times 10^{-4}$ to $100 \times 10^{-4}$. The low values at Sites $760(34$ $\left.\times 10^{-4}, 0-70 \mathrm{mbsf}\right)$ and $761\left(46 \times 10^{-4}, 0-230 \mathrm{mbsf}\right)$ suggest that calcite recrystallization has progressed to a more limited extent at Wombat Plateau sites than at Sites 762 and 763. Higher values at both Sites 762 and 763 (near $100 \times 10^{-4}$ ) attest to extensive recrystallization throughout the thick carbonate sequences. Based on the $\mathrm{Sr}$ profiles, recrystallization must begin within the oozes and continue well into the chalks at Sites 762 and 763 . Nonetheless, all $\delta \mathrm{Sr} / \delta \mathrm{Ca}$ ratios observed throughout Leg 122 sites are approximately two- to five-fold greater than the $\mathrm{Sr} / \mathrm{Ca}$ ratio of $\sim 20 \times 10^{-4}$ found in nannofossil oozes (Baker et al., 1982) and indicate that $\mathrm{Sr}$ is excluded during the recrystallization of calcite.

Large depletions of $\mathrm{Mg}$ downhole may be attributed partly to the formation of high magnesian calcites and dolomite, although uptake during the formation of smectite also contributes to the observed depletions (Kastner and Gieskes, 1976; Gieskes, 1981, 1985). Hence, carbonate and silicate diagenesis may be intimately related.

The negative $\mathrm{K}$ gradients of 1 to $3 \mathrm{mmol} / \mathrm{L} / 100 \mathrm{~m}$ observed in Leg 122 sediments are similar to the deep-sea drilling average of $2 \mathrm{mmol} / \mathrm{L} / 100 \mathrm{~m}$ noted by Gieskes (1981). However, the usual explanation that $\mathrm{K}$ is taken up during the alteration of volcanic matter and underlying igneous rocks seems inappropriate here because relatively little volcanic matter was found in Leg 122 sediments (Haq, von Rad, O'Connell, et al., 1990). Hence other processes such as the formation of K-feldspars in zones of silicification (Gieskes, 1985), or removal of K associated with the uptake of $\mathrm{Mg}$ in authigenic clays, must control the dissolved concentration of this element. An association of $\mathrm{K}$ uptake with dolomitization is unlikely.

Dissolved silica trends at Sites 761-763 are interpreted to represent (1) early dissolution of radiolarians near the sediment surface, (2) continued dissolution of opal-A, and (3) precipitation of opal-CT. Below the opal-CT region the decrease to $0.4-0.6 \mathrm{mM}$ represents the zone in which conversion of opal-CT to quartz occurs. Large silica fluctuations observed in the opal-CT zone have been described in terms of both variable and high dissolved silica concentrations remaining in sediments after opal-CT precipitation (Gieskes, 1981), and by local lithologic control on the silica solubility. Subsequent decreases in dissolved silica have also been reported to result from uptake by authigenic clays (Kastner and Gieskes, 1976), although a significant amount of the dissolved silica must go into the formation of authigenic quartz.

Lithium enrichments that generally occur immediately below the zone in which porewater silica concentrations decrease to less than $0.5 \mathrm{mM}$ (Fig. 3) are likely due to the exclusion of $\mathrm{Li}$ during recrystallization of opal-CT to quartz (Gieskes, 1981).

Early work by Lerman $(1975,1977)$ and subsequent studies by McDuff and colleagues (e.g., McDuff and Gieskes, 1976; McDuff et al., 1978; McDuff, 1981, 1985) have treated porewater profiles with simple advection/diffusion models. The models imply that nonlinear pore-water gradients are indicative of reaction zones. Without performing the calculations used in their models, the slight curvature of the $\mathrm{Ca}, \mathrm{K}$, and $\mathrm{Mg}$ profiles in Leg 122 sediments suggests that reaction zones occur within the sediments at Sites 759, 760, 762, and 763 . Even when the gradients appear linear, it is only so over limited intervals. Often the changes in gradient (i.e., their slope) correlate to changes in sediment lithology and porosity and arise from different rates of diffusion within the sediments. Hence the profiles may still be primarily diffusion 
profiles affected by a variable sediment porosity. Profiles of other constituents such as $\mathrm{Li}, \mathrm{Sr}$, silica, and sulfate display much more curved gradients, suggesting that these elements are extensively and actively involved in reactions within the sediment column. This is particularly true for silica; it is generally accepted that the high reactivity of this constituent is commonly sufficient to prevent the erasing of large concentrations gradients by diffusion.

In addition to the diffusive control on pore-water profiles, there is evidence of advective transport of freshwater in several Leg 122 sites. It is likely that a continued infusion of freshwater deep within Site 762 and 763 sediments contributes to the maintenance of significant negative $\mathrm{Cl}$ and salinity gradients. Such a process likely occurs, although to more a limited extent at the Wombat Plateau sites.

\section{ACKNOWLEDGMENTS}

I wish to express my appreciation to shipboard colleagues and to B. Haq, S. O'Connell, and U. von Rad for educating me in the ways of ODP, and providing a collegial working environment. Many thanks to Joe Powers and Matt Mefferd, and to Charles Fraley and Wayne Shibata, for technical assistance at sea and on shore, respectively. Discussions with Mike Mottl and Joris Gieskes helped me understand and interpret the data presented in this paper. Reviews by $\mathbf{J}$. Compton, S. O'Connell, and an anonymous reviewer helped significantly improve the manuscript. The financial research support provided by JOI/USSAC is deeply appreciated. This is SOEST contribution number 2403.

\section{REFERENCES}

Baker, P. A., Gieskes, J. M., and Elderfield, H., 1982. Diagenesis of carbonates in deep-sea sediments: evidence from $\mathrm{Sr}^{2+} / \mathrm{Ca}^{2+}$ ratios and interstitial dissolved $\mathrm{Sr}^{2+}$ data. J. Sediment. Petrol., 52:71-82.

Bischoff, J. L., Greer, R. E., and Luistro, A. O. 1970. Composition of interstitial waters of marine sediments: temperature of squeezing effect. Science, 167:1245-1246.

Bruland, K. W., 1983. Trace elements in seawater. In Riley, J. P., and Chester, R. (Eds.), Chemical Oceanography (Vol. 8): London (Academic Press), 157-220.

Couture, R., Miller, R. S., and Gieskes, J. M., 1977. Interstitial water and mineralogical studies, Leg 41. In Lancelot, Y., Seibold, E., et al., Init. Repts. DSDP, 41: Washington (U.S. Govt. Printing Office), 907-914.

Compton, J. S., in press. Early diagenesis and the origin of diagenetic carbonate in sediment recovered from the Argo Basin, northeastern Indian Ocean (Site 765). In Gradstein, F. M., Ludden, J. N., et al., Proc. ODP, Sci. Results, 123: College Station, TX (Ocean Drilling Program).

De Carlo, E. H., 1990. Ion-exchange chromatography and ICP/AES determination of lanthanide series elements in marine $\mathrm{Fe}-\mathrm{Mn}$ deposits. Sep. Sci. Tech., 35:781-798.

Emerson, S., Cranston, R. E., and Liss, P. S., 1979. Deep Sea Res. Part A, 26:859.

Exon, N. F., von Rad, U., and von Stackelberg, U., 1982. The geological evolution of the passive margins of the Exmouth Plateau off northwest Australia. Mar. Geol., 47:131-152.

Gieskes, J. M., 1974. Interstitial water studies, Leg 25. In Simpson, E.S.W., Schlich, R., et al., Init. Repts. DSDP, 25: Washington (U.S. Govt. Printing Office), 361-394.

1981. Deep-sea drilling interstitial water studies: implications for chemical alteration of the oceanic crust, layers I and II. In Warme, J. E., Douglas, R. G., and Winterer, E. L. (Eds.), The Deep Sea Drilling Project: A Decade of Progress. Soc. Econ. Paleontol. Mineral. Spec. Publ., 32:149-167.

1983. The chemistry of interstitial waters of deep-sea sediments: interpretation of Deep-Sea Drilling data. In Riley, J. P., and Chester, R. (Eds.), Chemical Oceanography (Vol. 8): London (Academic Press), 222-269.

Gieskes, J. M., Elderfield, H., Lawrence, J. R., Johnson, J., Meyers, B., and Campbell, A., 1982. Geochemistry of interstitial waters and sediments, Leg 64, Gulf of California. In Curray, J. R., Moore, D. G., et al., Init. Repts. DSDP, 64: Washington (U.S. Govt. Printing Office), 675-694.

Gieskes, J. M., Elderfield, H., and Nevsky, B., 1983. Interstitial water studies, Leg 65. In Lewis, B.T.R., Robinson, P., et al., Init. Repts. DSDP, 65: Washington (U.S. Govt. Printing Office), 441-449.

Gieskes, J. M., Lawrence, J. R., and Galleisky, G., 1978. Interstitial water studies, Leg 38. Init. Repts. DSDP, 38 (Suppl.): Washington (U.S. Govt. Printing Office), 121-123.

Gieskes, J. M., Lawrence, J. R., Perry, E. A., Grady, S. J., and Elderfield, H., 1987. Chemistry of interstitial waters and sediments in the Norwegian-Greenland sea, Deep Sea Drilling Project Leg 38. Chem. Geol., 63:143-155.

Gieskes, J. M., and Peretsman, G., 1986. Procedures of interstitial water analysis. ODP Tech. Note, 5.

Haq, B. U., von Rad, U., O'Connell, S., et al., 1990. Proc. ODP, Init. Repts., 122: College Station, TX (Ocean Drilling Program).

Harrison, W. E., Hesse, R., and Gieskes, J. M., 1982. Relationship between sedimentary facies and interstitial water chemistry of slope, trench, and Cocos plate sites from the Middle America Trench transect, active margin off Guatemala, Deep Sea Drilling Project Leg 67. In Aubouin, J., von Huene, R., et al., Init. Repts. DSDP, 67: Washington (U.S. Govt. Printing Office), 603-616.

Jenden, P. D., and Gieskes, J. M., 1983. Chemical and isotopic composition of interstitial water from Deep Sea Drilling Project Sites 533 and 534. In Sheridan, R. E., Gradstein, F. M., et al., Init. Repts. DSDP, 76: Washington (U.S. Govt. Printing Office), 453-461.

Kastner, M., and Gieskes, J. M., 1976. Interstitial water profiles and sites of diagenetic reactions, Leg 35, DSDP, Bellinghausen Abyssal Plain. Earth Planet. Sci. Lett., 33:11-20.

Kingston, H. M., and Jassie, L. B., 1988. Introduction to Microwave Sample Preparation: Am. Chem. Soc.

Lasaga, A. C., and Holland, H. D., 1976. Mathematical aspects of non-steady state diagenesis. Geochim. Cosmochim. Acta, 40:257266.

Lerman, A., 1975. Maintenance of steady state in oceanic sediments. Am. J. Sci., 275:609-635.

1977. Migrational processes and chemical reactions in interstitial waters. In Goldberg, E. D., and McCave, I. N. (Eds.), The Sea (Vol. 6): New York (Wiley), 695-738.

Ludden, J. N., Gradstein, F. M., et al., 1990. Proc. ODP, Init. Repts., 123: College Station, TX (Ocean Drilling Program).

Manheim, F. T., and Sayles, F. L., 1974. Composition and origin of interstitial waters of marine sediments based on deep sea drill cores. In Goldberg, E. D. (Ed.), The Sea (Vol. 5): New York (Wiley), 527-568.

Mann, R., and Gieskes, J. M., 1975. Interstitial water studies, Leg 28. In Hayes, D. E., Frakes, L. A., et al., Init. Repts. DSDP, 28: Washington (U.S. Govt. Printing Office), 805-814.

McDuff, R. E., 1981. Major cation gradients in DSDP interstitial waters: the role of diffusive exchange between seawater and upper oceanic crust. Geochim. Cosmochim. Acta, 45:1705-1713. 1985. The chemistry of interstitial waters, Deep Sea Drilling Project, Leg 86. In Heath, G. R., Burckle, L. H., et al., Init. Repts. DSDP, 86: Washington (U.S. Govt. Printing Office), 675-687.

McDuff, R. E., and Gieskes, J. M., 1976. Calcium and magnesium profiles in DSDP interstitial waters: diffusion or reaction? Earth Planet. Sci. Lett., 33:1-10.

McDuff, R. E., Gieskes, J. M., and Lawrence, J. R., 1978. Interstitial water studies, Leg 42A. In Hsü, K. J., Montadert, L., et al., Init. Repts. DSDP, 42 (Pt. 1): Washington (U.S. Govt. Printing Office), 561-568.

Riech, V., and von Rad, U., 1979. Silica diagenesis in the Atlantic Ocean: diagenetic potential and transformations. In Talwani, M., Hay, W., and Ryan, W.B.F. (Eds.), Deep Drilling Results in the Atlantic Ocean: Continental Margins and Paleoenvironment: Am. Geophys. Union, Maurice Ewing Ser., 3:315-340.

Schrader, H.-J., 1972. Kieselsaure-Skelette in sedimenten des iberomarok-kanischen Kontinentalrandes und angrendzender Tiefsee Ebenen. "Meteor" Forschungsergeb. Reihe C, 8:10-36.

Date of initial receipt: 29 May 1990

Date of acceptance: 2 March 1991

Ms 122B-129 